\title{
Chemical abundances for the outer halo cluster Pal 4 from co-added high-resolution spectroscopy ${ }^{\star}$
}

\author{
A. Koch ${ }^{1}$ and P. Côté \\ ${ }^{1}$ Department of Physics \& Astronomy, University of Leicester, University Road, Leicester LE1 7RH, UK \\ e-mail: ak326@astro.le.ac.uk \\ 2 National Research Council of Canada, Herzberg Institute of Astrophysics, 5071 West Saanich Road, Victoria, BC V9E 2E7, Canada \\ Received 29 January 2010 / Accepted 22 April 2010
}

\section{ABSTRACT}

\begin{abstract}
Context. Chemical element abundances for distant Galactic globular clusters (GCs) hold important clues to the origin of the Milky Way halo and its substructures.

Aims. We study the chemical composition of red giant stars in Pal 4 - one of the most remote GCs in the Milky Way - and compare our abundance measurements to those for both low surface brightness dwarf galaxies, and GCs in the inner and the outer halo.

Methods. By co-adding high-resolution, low-S/N Keck/HIRES spectra of 19 stars along the red giant branch, we estimate chemical abundance ratios of $20 \alpha$-, iron peak-, and neutron-capture elements. Our method gives total uncertainties on most element-to-iron ratios of typically 0.2 dex.

Results. We measure $[\mathrm{Fe} / \mathrm{H}]=-1.41 \pm 0.04$ (statistical) \pm 0.17 (systematic) and an $\alpha$-enhancement of $[\alpha / \mathrm{Fe}]=+0.38 \pm 0.11$ dex, which is consistent with the canonical value of $\sim+0.4$ dex found for Galactic halo field stars and most halo GCs at this metallicity. Although Pal 4 has higher enhancements in the heavier elements with respect to the halo, the majority of the element ratios are, within the measurement errors, consistent with those for local halo field stars. We find, however, evidence for a lower $[\mathrm{Mg} / \mathrm{Ca}] \mathrm{ratio}$ than in other halo clusters.

Conclusions. Based on the available evidence, we conclude that the material from which Pal 4 and the Galactic halo formed experienced similar enrichment processes, despite the apparently younger age of this cluster. Within the limitations of our methodology we find no significant indication of an iron spread, as is typical of genuine GCs of the Milky Way. However, abundance ratios for individual stars in Pal 4 and other distant satellites are urgently needed to understand the relationship, if any, between remote GCs and other halo substructures (i.e., luminous and ultra-faint dwarf spheroidal galaxies).
\end{abstract}

Key words. stars: abundances - Galaxy: abundances - Galaxy: evolution - Galaxy: halo - globular clusters: individual: Pal 4

\section{Introduction}

As the oldest readily identifiable stellar systems in the universe, globular clusters (GCs) are important tracers of the formation and early evolution of galaxies, the Milky Way (MW) included. Noting the apparent lack of a metallicity gradient among remote Galactic GCs, Searle \& Zinn (1978) proposed an accretion origin for the Galactic halo extending over a period of several Gyr. Evidence for this picture of hierarchical halo growth has come from the existence of a second-parameter problem among outer halo GCs (e.g., Catelan 2000; Dotter et al. 2010), which points to a significant age range within this population.

The remote GC Pal 4 is such an example of a second parameter cluster. At a Galactocentric distance of $R_{\mathrm{G}}=109 \mathrm{kpc}$ (Stetson et al. 1999), it is one of only a few halo GCs at distances of $\sim 100 \mathrm{kpc}$ or beyond. With a half-light radius of $r_{\mathrm{h}} \approx 23$ pc, it is also one of the most extended Galactic GCs currently known, being significantly larger than "typical" GCs in the Milky Way or external galaxies (which have $\left\langle r_{\mathrm{h}}\right\rangle \approx 3 \mathrm{pc}$; see, e.g., Jordán et al. 2005). In fact, with a total luminosity of just $L_{V} \sim 2.1 \times 10^{4} L_{V, \odot}$, it is similar in several respects to some of the more compact "ultra-faint" dwarf spheroidal (dSph) galaxies

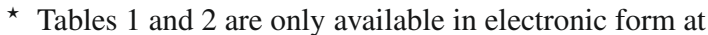
http://www . aanda.org
(Simon \& Geha 2007) that are being discovered in the outer halo with increasing regularity (e.g., Belokurov et al. 2007). Since almost nothing is known about their proper motions and internal dynamics, the relationship of faint, extended GCs like Pal 4 to such low-luminosity galaxies is currently an open question.

While there is a general consensus that Pal 4 is likely to be $\approx 1-2$ Gyr younger than inner halo GCs of the same metallicity, such as M 5, age estimates in the literature do not fully agree (e.g., Stetson et al. 1999; vs. Vandenberg 2000). In particular, Stetson et al. (1999) note that an age difference with respect to the inner halo GCs could be explained if "either $[\mathrm{Fe} / \mathrm{H}]$ or $[\alpha / \mathrm{Fe}]$ for the outer halo clusters is significantly lower than ... assumed". Conversely, Cohen \& Meléndez (2005a) found that the outer halo GC NGC $7492\left(R_{\mathrm{GC}}=25 \mathrm{kpc}\right)$ shows chemical abundance patterns that are very similar to inner halo GCs like M 3 or M 13. This similarity in the chemical enrichment now appears to extend into the outermost halo for at least some GCs: it has recently been shown that the abundance ratios in the remote $\left(R_{\mathrm{GC}}=92 \mathrm{kpc}\right)$ cluster Pal 3 (Koch et al. 2009, hereafter Paper I) bear a close resemblance to those of inner halo GCs. The chemical abundance patterns of remote halo GCs like Pal 3 and Pal 4 are important clues to the formation of the Milky Way, as they allow for direct comparisons to those of the dSph galaxies, which are widely believed to have been accreted into the halo (e.g., Klypin et al. 1999; Bullock et al. 2001; Font et al. 2006; 
Li et al. 2009). In this spirit, Mackey \& Gilmore (2004) conclude that all young halo clusters (i.e., 30) did not originate in the MW but were donated by at least seven mergers with "clusterbearing" dSph-type galaxies.

There are, however, no high-dispersion abundance data yet published for this remote cluster. Previous low-resolution spectroscopic and photometric studies have established Pal 4 as a mildly metal-poor system, with $[\mathrm{Fe} / \mathrm{H}]$ estimates ranging from -1.28 to -1.7 dex (Armandroff et al. 1992; Stetson et al. 1999; Kraft \& Ivans 2003). In this paper, one of a series, we aim to extend the chemical element information for GCs in the Galactic halo out to the largest possible distances, and to carry out a first analysis of Pal 4's chemical abundance patterns. As we have shown in Paper I, which presented a similar analysis for $\mathrm{Pal} 3$, it is possible to derive reliable abundance measurements for remote Galactic GCs by performing an integrated analysis of stacked, low signal-to-noise $(S / N)$ - but high-resolution - spectra (see also McWilliam \& Bernstein 2008). Note, however, that this method presupposes that there is no significant abundance scatter present along the RGB and that all stars have the same mean abundances for all chemical elements. We have therefore no means of distinguishing $\mathrm{Pal} 4$ as a genuine $\mathrm{GC}$ with no internal abundance spread from a dSph that may have a very broad abundance range (e.g., Shetrone et al. 2001, 2003; Koch 2009), nor of discerning any intrinsic abundance variations (e.g., Lee et al. 2009). We will return to this question in Sect. 5.2. Neverthess, such studies can provide an important first step towards an overall characterization of the chemical element distribution, and enrichment history, of the outer halo.

\section{Data}

The spectra for Pal 4 were obtained during the same three nights in February and March 1999 as the spectra used in our analysis of Pal 3 (Paper I). During these observing runs, a total of 24 stars in Pal 4 were observed using the HIRES echelle spectrograph (Vogt et al. 1994) on the Keck I telescope. Our spectroscopic targets were selected from a colour-magnitude diagram (CMD) constructed from $B V$ imaging obtained with the Low-Resolution Imaging Spectrometer (LRIS, Oke et al. 1995) on the night of 13/14 January 1999. A CMD reaching roughly one magnitude below the main-sequence turnoff was constructed using a series of short and long exposures taken in both bandpasses (i.e., five exposures between $60 \mathrm{~s}$ and $120 \mathrm{~s}$ in $V$, and four exposures between $60 \mathrm{~s}$ and $240 \mathrm{~s}$ in $B$ ).

Spectroscopic targets were identified from this CMD by selecting probable red giant branch (RGB) stars with $V \lesssim 20.25$. These stars all have cross identifications with the more recent work of Saha et al. (2005). Figure 1 shows the location of the target stars in the CMD from this latter work. As for Paper I, we used a spectrograph setting that covers the wavelength range 4450-6880 ^ with spectral gaps between adjacent orders, a slit width of $1.15^{\prime \prime}$ and a CCD binning of $2 \times 2$ in the spatial and spectral directions. This gives a spectral resolution of $R \approx 34000$. Each programme star was observed for a total of 300-2400 s, depending on its apparent magnitude (see Table 1; only available in electronic form). Table 1 also lists the photometric properties of the target stars, where the BVI photometry is taken from Saha et al. (2005) and the $K$-band magnitudes are from 2MASS (Skrutskie et al. 2006).

The spectroscopic data were reduced using the $\mathrm{MAKEE}^{1}$ data reduction package. Because our spectra were obtained

\footnotetext{
${ }^{1}$ MAKEE was developed by T. A. Barlow specifically for reduction of Keck HIRES data. It is freely available on the World Wide Web at
}

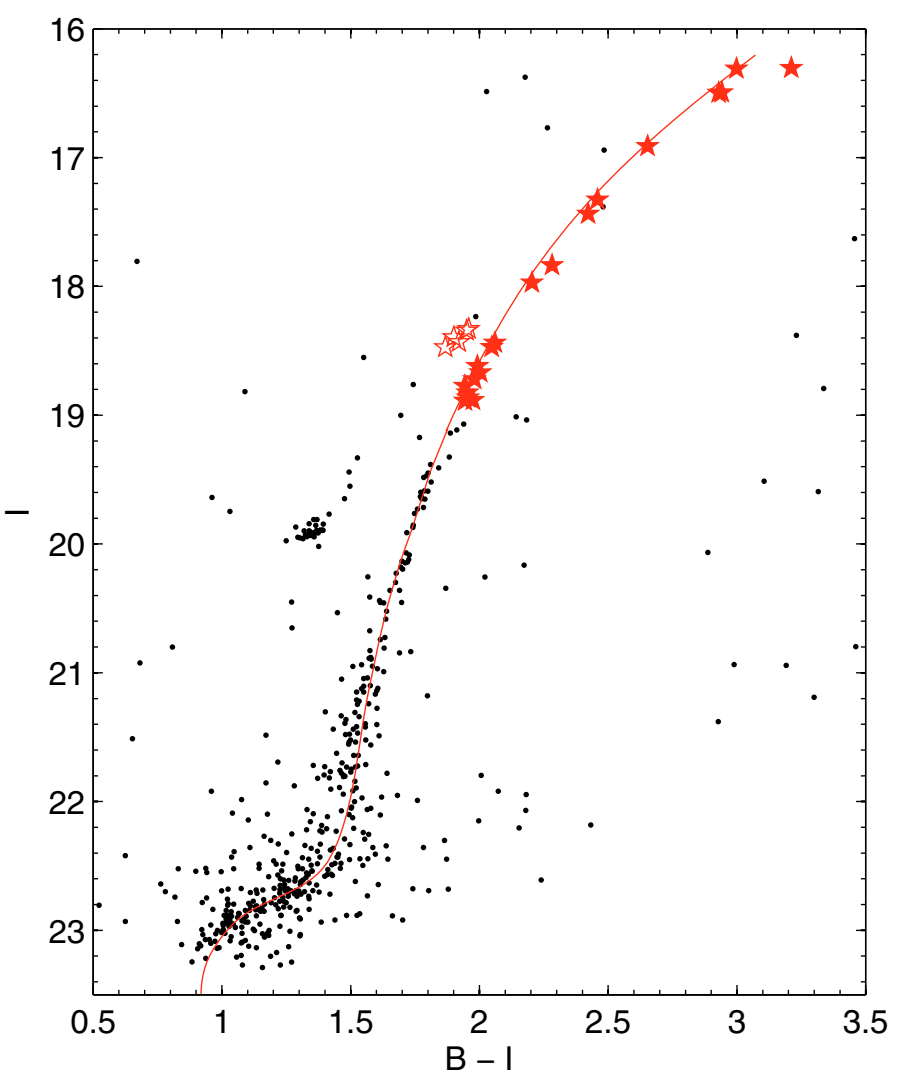

Fig. 1. CMD of Pal 4 based on photometry from Saha et al. (2005). The HIRES targets are highlighted as red symbols, with open stars denoting AGB candidates. Also shown is a scaled-solar Dartmouth isochrone (Dotter et al. 2008) with an age of $10 \mathrm{Gyr}$ and a $[\mathrm{Fe} / \mathrm{H}]$ of $-1.4 \mathrm{dex}$, corrected for $E(B-V)=0.01$ and a distance modulus of 20.22 mag (Stetson et al. 1999).

within a program to study the internal cluster dynamics (Côté et al. 2002; Jordi et al. 2009; Baumgardt et al. 2009), the short exposure times - which were chosen adaptively based on target magnitude - lead to low signal-to-noise $(S / N)$ ratios. Hence, the spectra are adequate for the measurement of accurate radial velocities but not for abundance measurements of individual stars. For instance, we typically reach $S / N$ ratios of 4-8 per pixel in the order containing $\mathrm{H} \alpha$.

Radial velocities of the individual targets were measured from a cross correlation against a synthetic red giant spectrum with stellar parameters representative of the Pal 4 target stars. The template covered the entire HIRES wavelength range, but excluded the spectral gaps. All our targets are consistent with the cluster's mean radial velocity of $\left\langle v_{\mathrm{r}}\right\rangle=72.9 \pm 0.3 \mathrm{~km} \mathrm{~s}^{-1}$ (mean error) within the measurement errors (see also Olszewski et al. 1986). A detailed account of the dynamics of Pal 4 will be given in a separate paper.

As in Paper I, we stack the individual spectra to increase their $S / N$ ratio and to be able to perform an integrated abundance analysis (see also McWilliam \& Bernstein 2008). In practice, the spectra were Doppler-shifted and average-combined after weighting by their individual $S / N$ ratios to yield a higher $S / N$ spectrum which we can use to place constraints on the chemical element abundances. As the CMD (Fig. 1) shows, five of the stars appear to lie on the AGB (open symbols). We therefore constructed three different co-added spectra: i.e., using only

the Keck Observatory home page, http: //www2 . keck . hawaii . edu/ inst/hires/makeewww 


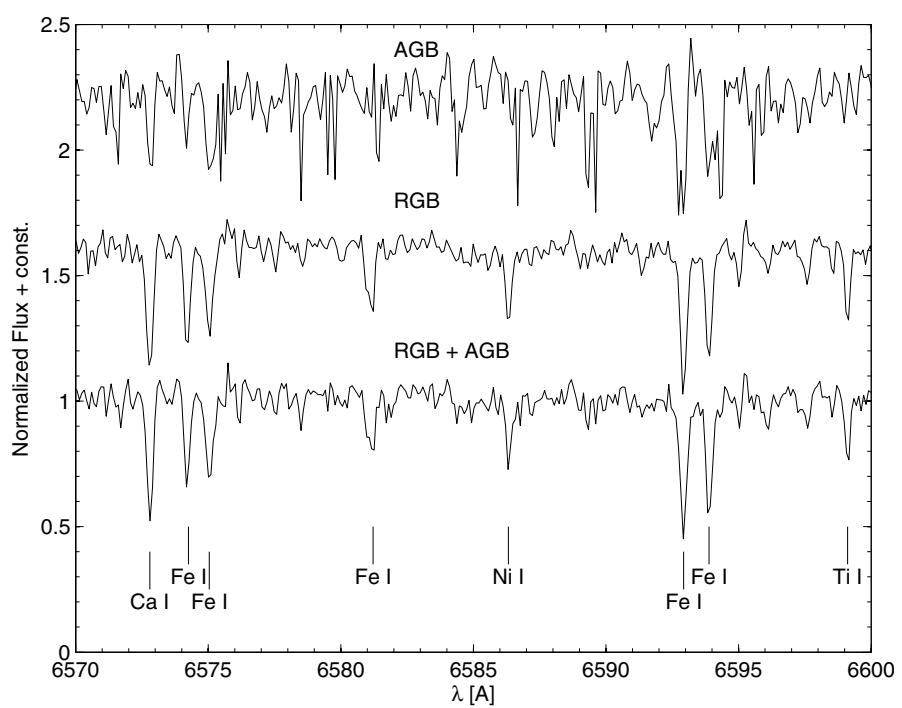

Fig. 2. A portion of the co-added spectra in one order with relatively high $S / N$ ratio. A few absorption lines are designated. Also indicated is the subsample of stars that was included in the co-additions.

the RGB stars, only the AGB stars, and the entire sample. The overall $S / N$ ratios of the co-added spectra are $(12,25,28)$ for the $\mathrm{AGB}, \mathrm{RGB}$, and the entire sample, respectively. A sample region of those spectra is shown in Fig. 2. It is obvious from this figure that the pure AGB spectrum still has a too low $S / N$ ratio for meaningful abundance measurements. Moreover, adding the AGB spectra to those of the higher- $S / N$ spectra for the RGB stars may introduce additional noise to some features rather than improving the spectral quality. We therefore choose focus our abundance analysis on the co-added RGB sample only.

\section{Abundance analysis}

As in our previous works (e.g., Paper I), we used model atmospheres interpolated from the updated grid of the Kurucz ${ }^{2}$ one-dimensional 72-layer, plane-parallel, line-blanketed models without convective overshoot and assuming local thermodynamic equilibrium (LTE) for all species. For this GC study, we used models that incorporate Castelli \& Kurucz's (2003) ${ }^{3}$ $\alpha$-enhanced opacity distribution functions, AODFNEW. This choice seems justified, since the majority of the metal-poor Galactic halo GCs, as well as the outer halo object Pal 3 (Paper I), are enhanced in the $\alpha$-elements by $\approx+0.4 \mathrm{dex}$, so it seems plausible that Pal 4 will also follow this trend. Throughout this work, we used the 2002 version of the stellar abundance code MOOG (Sneden 1973) for all abundance calculations. We place our measurements on the Solar scale of Asplund et al. (2009).

\subsection{Linelist}

We derive chemical element abundances through standard equivalent width $(E W)$ measurements that closely follow the procedures outlined in Paper I. The main difference is that we are only dealing with an analysis of the co-added $E W \mathrm{~s}$ in the present study, which thus requires an analogous treatment of the synthetic EWs. The linelist for the present study is the same as already used in Paper I and we refer the reader to that work for

\footnotetext{
2 http://cfaku5.cfa.harvard.edu/grids.html

3 http://wwwuser.oat.ts.astro.it/castelli
}

full details on the origin of the line data. In practice, we measured $E W$ s in the co-added spectra (Sect. 2) by fitting a Gaussian profile to the absorption lines using IRAF task splot; those value are recorded in Table 2 (online only).

Aided by the stellar atmospheres (described in detail in the next section), we computed theoretical $E W$ s for the transitions in our line list using MOOG's ewfind driver and combined them into a mean value, $\langle E W\rangle$, using the same weighting scheme as for the observations,

$$
\langle E W\rangle=\frac{\sum_{i=1}^{N} w_{i} E W_{i}}{\sum_{i=1}^{N} w_{i}},
$$

where the weights $w_{i}$ are proportional to the $S / N$ ratios as in the case of co-adding the observed spectra. The abundance ratio of each element was then varied until the predicted $\langle E W\rangle$ matched the observed $E W$ for each line to yield the cluster's integrated chemical element ratio. Note that this method presupposes that there is no significant abundance scatter present along the RGB and all stars have the same mean abundances for all chemical elements. For the following analysis, we restricted the linelist to the more reliable features with $E W<180 \mathrm{~m} \AA$. For a few cases, such as $\mathrm{Al}, \mathrm{Zr}, \mathrm{Ce}$, and Dy, the stated abundance ratios are based on marginal detections of only one line with usually about 30$40 \mathrm{~m} \AA$ widths. Unfortunately, neither of the important elements $\mathrm{O}$ and Eu could be detected: while the stronger [O I] $6300 \AA$ and Eu II $6645 \AA$ lines fall on the gap between the HIRES CCDs, the weaker $6363 \AA(\mathrm{O})$ and $6437 \AA$ (Eu) lines are strongly affected by telluric blends and spectral noise, which renders them unusable for the present work. Likewise, the Na-D lines are too strong to be reliably used in our analysis, while the only other transition covered by our spectra, the Na I $5688 \AA$ line, is too strongly affected by the low $S / N$ ratio around that feature.

We accounted for the effects of hyperfine structure for the stronger lines of the odd-Z elements Mn I, Co I, and $\mathrm{Cu}$ I by extracting the predicted $E W$ from MOOG's blends driver and using atomic data for the splitting from McWilliam et al. (1995). The effect on all other elements (such as Ba II or La II) was found to be typically less than 0.03 dex and thus much smaller than the usual systematic errors (Sect. 4) so that we ignored hyperfine splitting for all other elements.

\subsection{Stellar parameters}

We derived effective temperatures $\left(T_{\text {eff }}\right)$ for each star using its photometry, in particular, from $B-V$ and $V-I$ colors using the data from Saha et al. (2005). This information was supplemented with 2MASS $K$-band photometry (Skrutskie et al. 2006) to obtain $V-K$ estimates for the eight brightest stars (see Table 1). We assumed a reddening of $E(B-V)=0.01$ (Stetson et al. 1999) with the extinction law of Winkler (1997). In practice, the $T_{\text {eff }}{ }^{-}$ color calibrations of Ramírez \& Meléndez (2005) were applied for $V-I$ and $V-K$, and the Alonso et al. (1999) transformations for $B-V$. All three values agree well with offsets of 6 and $20 \mathrm{~K}$ for $B-V$ vs. $V-I$ and $V-K$, with an rms scatter of 50 and $80 \mathrm{~K}$, respectively. For all these calibrations, we adopted the cluster mean metallicity of -1.43 dex on the Kraft \& Ivans (2003) scale. The resulting temperatures have a formal mean random error due to color and calibration uncertainties of $136 \mathrm{~K}$ on average. In practice, we adopt an error-weighted mean of all three color indicators as the final $T_{\text {eff }}$ for the atmospheres. Figure 3 shows the distribution of effective temperatures for our targets. 


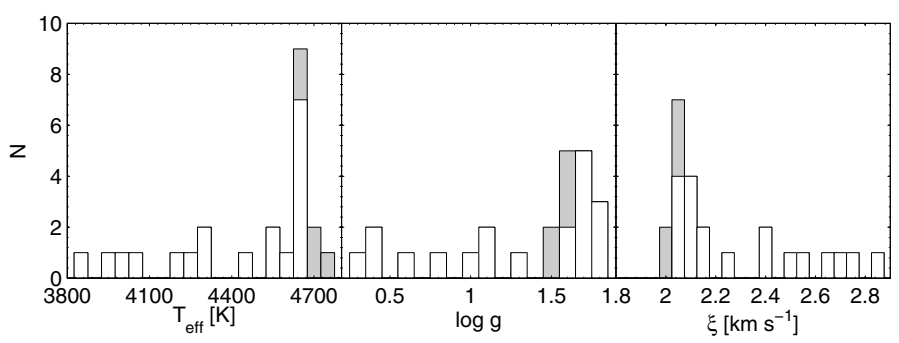

Fig. 3. Distribution of stellar parameters for the RBG and AGB stars (open and shaded histograms, respectively).

Surface gravities, $\log g$, were derived from the photometry, an adopted distance modulus of 20.22 mag (Stetson et al. 1999), and the above temperature and metallicity estimates. A mass of $0.85 M_{\odot}$ was adopted for the red giants, as indicated by a comparison with the Dartmouth isochrones (Dotter et al. 2008; Fig. 1). Errors on the input parameters (predominantly that on $T_{\text {eff }}$ ) lead to a typical uncertainty in $\log g$ of \pm 0.16 dex. As in Paper I, we derived microturbulent velocities, $\xi$, from a linear fit to the temperatures of halo stars that have similar parameters to ours (Cayrel et al. 2004). The scatter around the best-fit relation implies a typical error of $\sigma(\xi) \approx 0.25 \mathrm{~km} \mathrm{~s}^{-1}$.

Since we have no prior knowledge of the individual stellar metallicities, we adopt the value of -1.43 dex (Kraft \& Ivans 2003) as representative of the cluster mean and as an input metallicity for the atmospheres. This value is then refined iteratively using the Fe I abundance from the previous step as input for the following atmosphere calculations.

In addition, we calculate an independent metallicity estimate for individual stars from the $\mathrm{Mg} \mathrm{I}$ line index at 5167, $5173 \AA$, which is defined and calibrated on the scale of Carretta \& Gratton (1997) as in Walker et al. (2007) and Eq. (2) in Paper I. For this, we assume a horizontal branch magnitude, $V_{\mathrm{HB}}$, of 20.8 mag (Stetson et al. 1999). Although we list the Mg I indicator in our final abundance in Table 3, we emphasize that this value is meant as an initial estimate of the cluster metallicity, rather than a reliable measurement of its abundance scale. Table 3 lists the final abundance ratios derived from the co-added red giant sample. Here, neutral species are given relative to Fe I, while the ratios of ionized species are listed with respect to the ionized iron abundance as [X II/Fe II].

\section{Abundance errors}

As a measure of the random uncertainties on our abundance ratios, Table 3 also lists the $1 \sigma$-scatter of the line-by-line measurements together with the number of transitions, $N$, used in the analysis. This contribution is generally small for those species with many suitable transitions (e.g., Fe I, Ca, Ti I, Ni) yet dominates for the other, poorly-sampled elements. As in Paper I, we adopt in what follows a minimum random abundance error of 0.10 dex and assign an uncertainty of 0.15 dex if only one line could be measured.

In order to investigate the extent to which inaccurate radial velocity measurements can lead to a broadening of the observed lines during the co-addition of the individual, Doppler-shifted spectra, we carried out a series of 1000 Monte Carlo simulations. In each simulation, we corrected every spectrum by a velocity that accounted for the velocity error before combining those falsified spectra into a new spectrum. The $E W$ s for the entire line list were then re-measured from each of those spectra in an
Table 3. Abundance results from the co-added red giant spectrum.

\begin{tabular}{crcrc}
\hline \hline Element & {$[\mathrm{X} / \mathrm{Fe}]$} & $\sigma$ & $N$ & $\sigma_{\text {tot }}$ \\
\hline $\mathrm{Fe}_{\mathrm{MgI}}{ }^{a}$ & -1.41 & 0.28 & $\ldots$ & $\ldots$ \\
$\mathrm{Fe}$ I & -1.41 & 0.35 & 81 & 0.17 \\
$\mathrm{Fe} \mathrm{II}$ & -1.54 & 0.21 & 8 & 0.25 \\
$\mathrm{Mg}$ I & 0.25 & 0.21 & 2 & 0.20 \\
$\mathrm{Al} \mathrm{I}$ & 0.36 & $\ldots$ & 1 & 0.19 \\
$\mathrm{Si} \mathrm{I}$ & 0.47 & 0.32 & 5 & 0.15 \\
$\mathrm{Ca}$ I & 0.40 & 0.16 & 11 & 0.21 \\
$\mathrm{Sc} \mathrm{II}$ & 0.29 & 0.28 & 6 & 0.14 \\
$\mathrm{Ti} \mathrm{I}$ & 0.24 & 0.32 & 18 & 0.30 \\
$\mathrm{Ti}$ II & 0.61 & 0.39 & 8 & 0.17 \\
$\mathrm{~V} \mathrm{I}$ & 0.16 & 0.17 & 6 & 0.29 \\
$\mathrm{Cr}$ I & -0.18 & 0.19 & 7 & 0.28 \\
$\mathrm{Mn} \mathrm{I}$ & -0.18 & 0.20 & 5 & 0.23 \\
$\mathrm{Co} \mathrm{I}$ & 0.38 & 0.07 & 3 & 0.18 \\
$\mathrm{Ni} \mathrm{I}$ & 0.04 & 0.29 & 16 & 0.14 \\
$\mathrm{Cu}$ I & -0.66 & 0.16 & 2 & 0.18 \\
$\mathrm{Y} \mathrm{II}$ & 0.30 & 0.29 & 5 & 0.17 \\
$\mathrm{Zr} \mathrm{II}$ & 0.53 & $\ldots$ & 1 & 0.16 \\
$\mathrm{Ba}$ II & 0.36 & 0.16 & 4 & 0.19 \\
$\mathrm{La} \mathrm{II}$ & 0.67 & 0.10 & 2 & 0.12 \\
$\mathrm{Ce}$ II & 0.34 & $\ldots$ & 1 & 0.16 \\
$\mathrm{Nd} \mathrm{II}$ & 0.45 & $\ldots$ & 1 & 0.16 \\
$\mathrm{Dy}$ II & 0.32 & $\ldots$ & 1 & 0.16 \\
\hline
\end{tabular}

Notes. ${ }^{(a)}$ Metallicity estimate based on the Mg I calibration of Walker et al. (2007), on the metallicity scale of Carretta \& Gratton (1997).

automated manner. As a result, the EWs changed by $(10 \pm 5) \%$ on average, with $1 \sigma$ of the widths changing by less than $15 \%$. We then repeated our abundance determinations by Monte Carlo varying the $E W$ s by this amount and deriving new means and dispersions. This revealed that a $15 \%$ uncertainty in the measured $E W$ incurs an error of 0.04 dex on the mean iron abundance. For this, we conclude that inaccurate Doppler-shifts of the spectra are not a major source of uncertainty in an analysis of this sort. The main contributor to the random errors are instead the $E W$ measurements at these still-low $S / N$ levels and, to a lesser extent, the standard uncertainties in the atmosphere models and atomic parameters themselves.

Although none of our stars is a likely non-member in terms of our CMD selection, nor indicated by deviating gravity sensitive features as the $\mathrm{Mg} b$ triplet or the $\mathrm{Na}-\mathrm{D}$ lines, nor by discrepant radial velocity, we explored the effect of co-adding undesired foreground dwarfs to the red giant sample on the resulting abundance ratios. To this end, we computed synthetic spectra for each star, using the atmospheric parameters determined above and adopting the element ratios listed in Table 3 . We then synthesized a spectrum of a metal-poor dwarf $\operatorname{star}\left(T_{\text {eff }}=5700 \mathrm{~K}\right.$, $\log g=4.2$, and $\xi=1.1 \mathrm{~km} \mathrm{~s}^{-1}$ ) and randomly replaced one or two of the RGB stars with a dwarf spectrum in the co-addition. The EWs of the resulting, co-added synthetic spectrum were then re-measured as above. As a consequence, the presence of one (two) underlying dwarf stars in the co-added spectrum does not change the co-added $E W \mathrm{~s}$ by more than $5 \%$ (9\%), on average. Thus our abundance ratios are insensitive to any residual foreground contamination, with no expected effect larger than 0.02 dex.

Systematic uncertainties of the stellar parameters were evaluated from a standard error analysis (e.g., Koch \& McWilliam 2010). To this end, each parameter was varied by the typical 
Table 4. Error analysis: deviations from the abundances in Table 3.

\begin{tabular}{|c|c|c|c|c|}
\hline Ion & $\begin{array}{c}\Delta T_{\text {eff }} \\
\pm 150 \mathrm{~K}\end{array}$ & $\begin{array}{l}\Delta \log g \\
\pm 0.2 \mathrm{dex}\end{array}$ & $\begin{array}{c}\Delta \xi \\
\pm 0.25 \mathrm{~km} \mathrm{~s}^{-1}\end{array}$ & ODF \\
\hline $\mathrm{Fe} \mathrm{I}$ & \pm 0.13 & \pm 0.01 & $\mp 0.12$ & -0.02 \\
\hline $\mathrm{Fe}$ II & $\mp 0.20$ & \pm 0.12 & $\mp 0.09$ & -0.13 \\
\hline Mg I & \pm 0.10 & $\mp 0.02$ & $\mp 0.10$ & 0.02 \\
\hline Al I & \pm 0.13 & $\mp 0.02$ & $\mp 0.01$ & 0.04 \\
\hline Si I & $\mp 0.03$ & \pm 0.03 & $\mp 0.03$ & -0.02 \\
\hline $\mathrm{Ca} \mathrm{I}$ & \pm 0.19 & $\mp 0.03$ & $\mp 0.08$ & 0.03 \\
\hline $\mathrm{Sc}$ II & $\mp 0.02$ & \pm 0.07 & $\mp 0.05$ & 0.04 \\
\hline Ti I & \pm 0.30 & $\mp 0.03$ & $\mp 0.08$ & 0.01 \\
\hline Ti II & $\mp 0.03$ & \pm 0.07 & $\mp 0.08$ & 0.04 \\
\hline V I & \pm 0.32 & $\mp 0.02$ & $\mp 0.03$ & 0.00 \\
\hline Cr I & \pm 0.26 & $\mp 0.03$ & $\mp 0.10$ & 0.02 \\
\hline Mn I & \pm 0.22 & \pm 0.01 & \pm 0.04 & 0.04 \\
\hline Co I & \pm 0.16 & \pm 0.03 & \pm 0.03 & 0.01 \\
\hline Ni I & \pm 0.11 & \pm 0.01 & $\mp 0.06$ & -0.01 \\
\hline $\mathrm{Cu} \mathrm{I}$ & \pm 0.15 & \pm 0.02 & $\mp 0.03$ & 0.01 \\
\hline Y II & \pm 0.01 & \pm 0.06 & $\mp 0.10$ & 0.04 \\
\hline Zr II & $\mp 0.02$ & \pm 0.07 & $\mp 0.02$ & 0.05 \\
\hline Ba II & \pm 0.05 & \pm 0.06 & $\mp 0.15$ & 0.01 \\
\hline La II & \pm 0.04 & \pm 0.07 & $\mp 0.02$ & 0.03 \\
\hline Ce II & \pm 0.02 & \pm 0.07 & $\mp 0.01$ & 0.04 \\
\hline Nd II & \pm 0.02 & \pm 0.06 & $\mp 0.03$ & 0.04 \\
\hline Dy II & \pm 0.05 & \pm 0.06 & \pm 0.00 & 0.04 \\
\hline
\end{tabular}

uncertainty ( $T_{\text {eff }} \pm 150 \mathrm{~K} ; \log g \pm 0.2 \mathrm{dex} ; \xi \pm 0.25 \mathrm{~km} \mathrm{~s}^{-1}$; see previous section), from which new atmospheres were interpolated for each star. This assumes that all stars are systematically affected in the same manner by the same absolute error. Furthermore, the column labeled "ODF" shows the changes induced by using the Solar-scaled opacity distributions ODFNEW, which corresponds to an error in the $\alpha$-enhancement of 0.4 dex. Using these changed atmospheres, theoretical $E W \mathrm{~s}$ were computed for each star and then combined into a new $\langle E W\rangle$ to be compared with the observed $E W$ as before. We list in Table 4 the deviations of the resulting new abundances from the nominal values, $[\mathrm{X} / \mathrm{Fe}]$, obtained from the unchanged atmospheres. Overall, the largest effect is naturally found with regard to $T_{\text {eff }}$ errors, while changes in $\log g$ mostly affect the ionized species (see also Paper I).

Finally, we interpolated the values in Table 4 to the actual parameter uncertainties estimated in Sect. 3.2 and adopted an error of the atmosphere $\alpha$-enhancement of \pm 0.2 (in accordance with the results for $[\alpha / \mathrm{Fe}]$ in Table 3$)$. These contributions were added in quadrature to the random error to yield the total abundance error, which we list as $\sigma_{\text {tot }}$ in the last column of Table 3 and which we will show in the following figures unless noted otherwise. Since this procedure neglects the covariances between the stellar parameters, these errors can be regarded as upper limits on the actual abundance uncertainties. In the end, our measurements yield element ratios that are typically accurate to within 0.2 dex for the $\alpha$-elements, $0.15-0.30$ dex for the iron peak elements, and approximately 0.2 dex for the heavy elements. Although these error estimates may seem relatively large (and dominated by the systematic uncertainties), we have shown in Paper I that the results from a co-added abundance analysis of this kind are largely consistent with those obtained from individual, high- $S / N$ spectroscopic measurements. Thus, the present data are adequate for placing useful limits on the chemical abundances in $\mathrm{Pal} 4$ and characterizing the general trends (see also Shetrone et al. 2009).

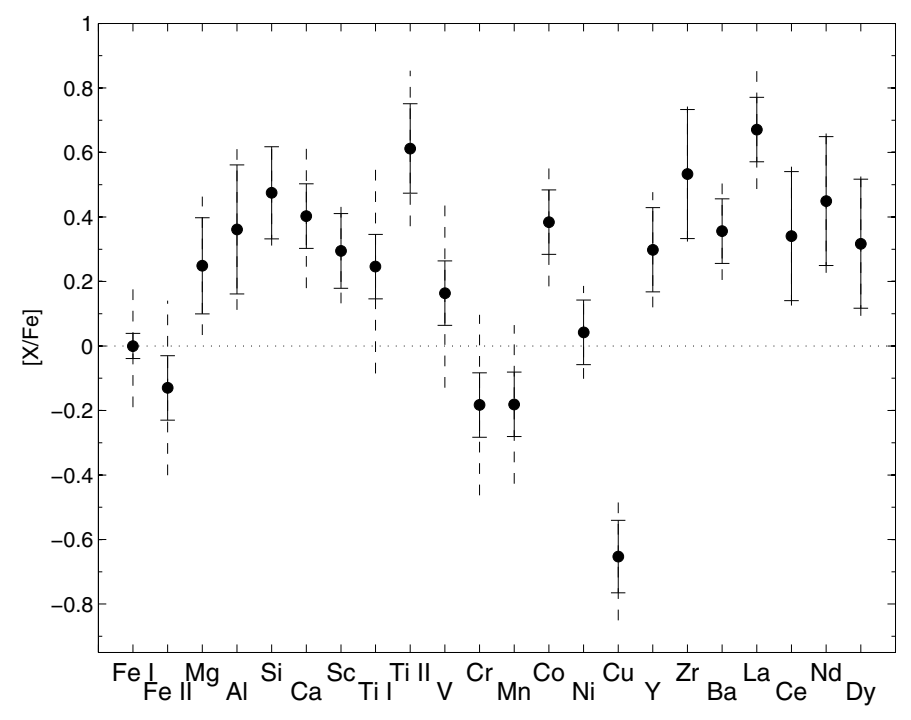

Fig. 4. Abundances ratios from the co-added RGB star spectrum. The dashed error bars indicate the total uncertainties (Tables 3 in 4), while the solid symbols represent $1 \sigma$ random errors.

\section{Abundance results}

Our abundance measurements based on the co-added RGB spectrum are plotted in Fig. 4. Note that the values for [Al, $\mathrm{Zr}, \mathrm{Ce}$, $\mathrm{Dy} / \mathrm{Fe}$ ] are only upper limits (Sect. 3.1), although we show their formal, total error bars in this figure (cf. Fig. 9).

\subsection{Iron}

Based on our sample of 19 RGB stars, we find a mean iron abundance of

$$
[\mathrm{FeI} / \mathrm{H}]=-1.41 \pm 0.04(\text { statistical }) \pm 0.17 \text { (systematic) } .
$$

This value is in excellent agreement with the Fe II based abundance scale of Kraft \& Ivans (2003), and slightly more metalpoor than the value of $-1.28 \pm 0.20$ dex reported by Armandroff et al. (1992) from the calcium triplet on the Zinn \& West (1984) scale, and by Stetson et al. (1999) from photometry. It is interesting to note that also the mean $[\mathrm{Fe} / \mathrm{H}]_{\mathrm{Mg} \text { I }}$ from the $\mathrm{Mg} b$ index (Sect. 3.2) agrees very well with the Fe I scale: for the red giant sample we find the same mean of $-1.41 \mathrm{dex}$, with a $1 \sigma$ spread of 0.28 dex.

Ionization equilibrium is not fulfilled in this integrated analysis to within the random uncertainties, while both stages agree if one accounts for their total errors; the mean deviation of the neutral and ionized species is $[\mathrm{Fe} \mathrm{I} / \mathrm{Fe} \mathrm{II}]=0.13 \pm 0.08 \mathrm{dex}$. A similar deviation was found in an identical analysis of co-addded RGB star spectra in Paper I, although in the opposite sense (i.e., with Fe I yielding higher abundances). As in Paper I, we conclude that Fe II lines in general seem ill suited to establishing a population's iron abundance from a low- $S / N$ spectral co-addition (cf. Kraft \& Ivans 2003; McWilliam \& Bernstein 2008). Typical $E W \mathrm{~s}$ of the eight Fe II lines we used in the analysis fall in the range 20-90 $\mathrm{m} \AA$. As Table 4 indicates, a systematic increase of 0.24 dex in the surface gravity would settle the ionization equilibrium at $[\mathrm{Fe} / \mathrm{H}]$ of $-1.39 \mathrm{dex}$, which is entirely consistent with the value found above from the neutral species. Moreover, a change in the temperature scale of just $-54 \mathrm{~K}$ (without altering $\log g$ ) would re-install the equilibrium at -1.44 dex (see also 
Koch \& McWilliam 2010). In what follows, we therefore proceed with our adopted $\log g$ scale and take the imbalance between ionized and neutral species at face value.

\subsection{Tests for abundance spreads}

As argued earlier, an integrated abundance analysis works reliably under the ad-hoc assumption of the same chemical abundance for all stars that enter the co-added spectrum. Here we discuss several tests of how realistic this assumption is for our analysis of Pal 4.

As a first test, we consider the spread in colour about the fiducial isochrone shown in Fig. 1. By interpolating a finely spaced isochrone grid in metallicity and using the identical values for age, distance modulus, and reddening as above, we find that the colour range of the RGB targets translates into a metallicity spread of 0.036 dex. Accounting for photometric errors, which propagate to a mean metallicity error of 0.026 dex, we find an intrinsic spread of $0.025 \mathrm{dex}$ in the photometric metallicities. Since this procedure did not include errors on the distance modulus or reddening, and uncertainties in the adopted age and $\alpha$-enhancement of the isochrones will lead to even larger uncertainties, we conclude that there is no evidence of any global abundance spread on the RGB, based on the photometric metallicities alone. This notion is consistent with the homogeneity (in iron or overall metallicity) of most genuine Galactic GCs (e.g., Carretta et al. 2009).

Secondly, we divided the RGB sample in two halves and coadded spectra for each subsample ${ }^{4}$. The above procedures to obtain iron abundance constraints from the co-added $E W$ s were repeated and we find slightly more metal poor values for either subsample: $-1.46 \pm 0.05$ and $-1.45 \pm 0.06$ dex, respectively, where the stated uncertainties account for random errors only. Therefore, there is no evidence of an abundance difference between the subsets within the measurement errors. Strictly, one would need to repeat this exercise for all (92378) possible combinations in order to detect the maximum abundance difference, which could be indicative of any real spread. The measurement of the $81 \mathrm{Fe}$ lines in this amount of spectra is, however, computational expensive and beyond our present scope.

As a last test, we employed a line-coaddition technique within the spectrum of each individual star, similar to that outlined in Norris et al. (2007, and references therein); see also Koch et al. (2008c): for each star, the useable $81 \mathrm{Fe}$ lines were thus shifted to zero wavelength at each line center, and then coadded into a composite, "master line". The same was carried out for a synthetic spectrum that matches the stellar parameters of the stars. This way we find a $1 \sigma$ dispersion of the $19[\mathrm{Fe} / \mathrm{H}]$ values of $0.176 \mathrm{dex}$. If we account for the random measurement errors from this procedure and assume the same systematic uncertainties as in our proper analysis (Sect. 4), we estimate an intrinsic abundance spread of no more than 0.05 dex. This is most likely an upper limit, since radial velocity uncertainties may have a larger impact on this method, and it is also not self evident that the systematic errors are identical to those in Table 3. At this low internal dispersion, however, Pal 4 does not comply with the broad ranges found in the dSphs (e.g., Table 1 in Koch 2009), while it is consistent with the upper limit for GC homogeneity found in Carretta et al. (2009). We conclude that, within the limitations of our spectral co-addition techniques, Pal 4 most

\footnotetext{
4 In practice stars were chosen to alternate in magnitude so that sample \#1 includes Pal4-1,3,6,8,10,19,23,25,28,31, and the remainder constitutes sample \#2.
}

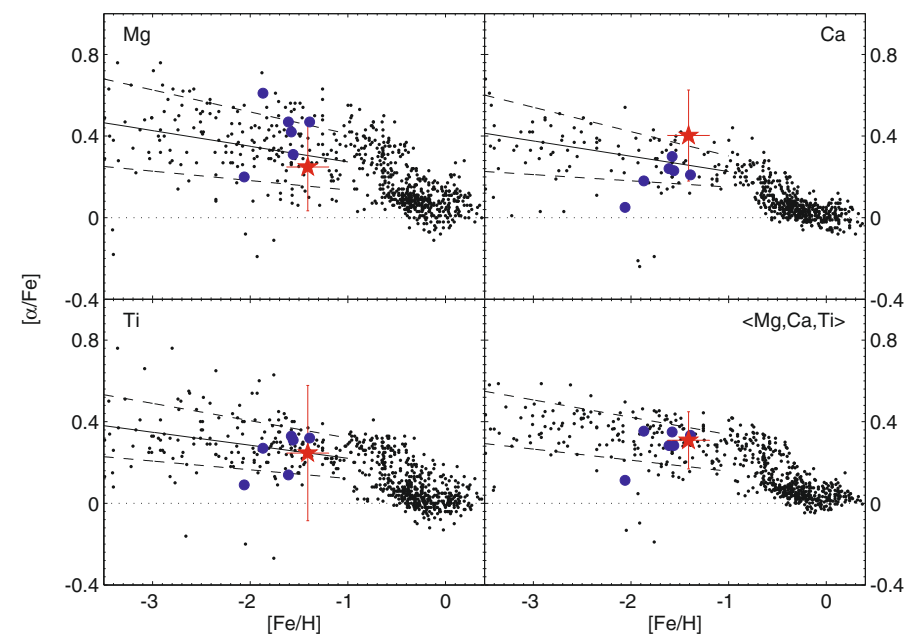

Fig. 5. $[\alpha / \mathrm{Fe}]$ abundance ratios for Pal 4 from this work (filled red star) in comparison with the GCs listed in Table 5 (solid dark blue circles). The solid and dashed lines illustrate the mean star relation and its $\pm 1 \sigma$ spread, respectively, from linear fits to the halo star data (black dots). See text for details.

likely shows little to no abundance spread, rendering it a genuine (MW) GC and arguing against an origin in a dSph-like environment.

\subsection{Alpha-elements}

All $\alpha$-elements measured in this study are enhanced with respect to $\mathrm{Fe}$. While the $[\mathrm{Ca} / \mathrm{Fe}]$ and $[\mathrm{Si} / \mathrm{Fe}]$ ratios show the canonical value of $\gtrsim 0.4$ dex typical for Galactic halo field and GC stars, the abundance ratios of $\mathrm{Mg}$ and $\mathrm{Ti}$ are slightly lower, at about 0.25 dex. Because the latter species have slightly larger errors, the error-weighted mean of all four elements is

$[\alpha / \mathrm{Fe}]=0.38 \pm 0.11 \mathrm{dex}$.

The $\alpha$-element ratios are shown for $\mathrm{Mg}, \mathrm{Ca}$, and $\mathrm{Ti}$ in Fig. 5 where they are compared to Galactic halo and disk data from the literature (small black dots). The data shown here are taken from the same sources as in Paper I. At this point, we draw the reader's attention to an important caveat in Fig. 5 and subsequent figures: the selection of halo stars used in these comparisons is, by necessity, a local sample. How appropriate it is to use local halo field stars in a comparison to remote halo GCs is unclear, particularly if there are radial gradients in the abundance ratios, as has sometimes been claimed (e.g., Nissen \& Schuster 1997; Fulbright 2002). We shall return in Sect. 5.6 to the issue of $\alpha$-element enhancements amongst different populations in the Galactic halo.

We note in passing that, although the difference $[\mathrm{Ti} \mathrm{I} / \mathrm{Ti} \mathrm{II}]=$ -0.24 dex is large, ionization equilibrium for $\mathrm{Ti}$ is satisfied considering the large combined total error for both species. This discrepancy is only significant at the $0.6 \sigma$-level and is in the opposite sense of the deviation in Fe. In any case, a detailed interpretation of any imbalances in terms of cumulative nonLTE effects along the RGB in our integrated abundance analysis would be beyond the scope of the present work (e.g., Koch \& McWilliam 2010).

At $-0.15 \mathrm{dex}$, the $[\mathrm{Mg} / \mathrm{Ca}]$ ratio is comparably low. While $\mathrm{Mg}$ is produced during the hydrostatic burning phases in the type II supernova (SN) progenitors, Ca nucleosynthesis proceeds during the SN explosion itself (e.g., Woosley \& Weaver 1995). 
Table 5. Pal 4 abundances relative to comparison clusters.

\begin{tabular}{rcccccccc}
\hline \hline Name & {$[\mathrm{Fe} / \mathrm{H}]$} & $R_{\mathrm{GC}}[\mathrm{kpc}]$ & $N$ & $\langle\Delta[\mathrm{X} / \mathrm{Fe}]\rangle_{\text {all }}$ & $\langle\Delta[\mathrm{X} / \mathrm{Fe}]\rangle_{Z<39}$ & $\langle\Delta[\mathrm{X} / \mathrm{Fe}] / \sigma\rangle_{\text {all }}$ & $\langle\Delta[\mathrm{X} / \mathrm{Fe}] / \sigma\rangle_{Z<39}$ & Reference $^{a}$ \\
$(1)$ & $(2)$ & $(3)$ & $(4)$ & $(5)$ & $(6)$ & $(7)$ & $(8)$ & $(9)$ \\
\hline NGC 6752 & -1.61 & 5.2 & 17 & $0.18 \pm 0.06$ & $0.10 \pm 0.08$ & 1.0 & 0.5 & $(1),(2)$ \\
M 13 & -1.56 & 8.7 & 17 & $0.15 \pm 0.06$ & $0.04 \pm 0.06$ & 0.8 & 0.2 & $(1),(3)$ \\
M 3 & -1.39 & 12.3 & 18 & $0.09 \pm 0.06$ & $-0.01 \pm 0.08$ & 0.5 & 0.0 & $(1),(3)$ \\
NGC 7492 & -1.87 & 24.9 & 16 & $0.09 \pm 0.05$ & $0.02 \pm 0.05$ & 0.4 & 0.1 & $(1),(4)$ \\
NGC 5694 & -2.06 & 29.1 & 10 & $0.36 \pm 0.12$ & $0.14 \pm 0.06$ & 1.8 & 0.6 & $(1),(5)$ \\
Pal 3 & -1.58 & 95.9 & 19 & $0.05 \pm 0.05$ & $-0.05 \pm 0.06$ & 0.3 & -0.1 & $(1),(6)$ \\
\hline
\end{tabular}

Notes. ${ }^{(a)}$ (1) Web-version (2003) of Harris (1996); (2) Yong et al. (2005); (3) Cohen \& Meléndez (2005b); (4) Cohen \& Meléndez (2005a); (5) Lee et al. (2006); (6) Paper I.

Thus, it is not evident that one element should trace the other over a broad metallicity range. In fact, theoretical yields predict a delicate mass dependance of the $[\mathrm{Mg} / \mathrm{Ca}]$ ratio. In Fig. 6, we show the distributions of this ratio for Galactic halo stars (gray shaded histogram) using the data of Gratton \& Sneden (1988, 1994), McWilliam et al. (1995), Ryan et al. (1996), Nissen \& Schuster (1997), McWilliam (1998), Hanson et al. (1998), Burris et al. (2000), Fulbright (2000, 2002), Stephens \& Boesgaard (2002), Johnson (2002), Ivans et al. (2003) and Cayrel et al. (2004).

Figure 6 also shows the currently available measurements for Local Group dSph galaxies (black line in Fig. 6) by Shetrone et al. (2001, 2003, 2009), Sadakane et al. (2004), Monaco et al. (2005), Letarte (2007), Koch et al. (2008a,b), Frebel et al. (2010), Aoki et al. (2009), Cohen \& Huang (2009) and Feltzing et al. (2009); see also Koch (2009). Halo stars scatter around a $[\mathrm{Mg} / \mathrm{Ca}]$ of zero, with mean and $1 \sigma$ dispersion of 0.05 and 0.15 dex, respectively. Stars with very low abundance ratios are the exception (e.g., Lai et al. 2009). In fact, the third moment of the halo distribution, at +0.55 , indicates a higher- $[\mathrm{Mg} / \mathrm{Ca}]$ tail. The dSph galaxies, on the other hand, have a formal mean and dispersion of 0.12 and 0.23 dex. It is important to bear in mind, though, that the abundance ratios in the dSphs are inevitably unique characteristics of each galaxy and should be governed by their individual star formation histories and global properties (e.g., Lanfranchi \& Matteucci 2004). In particular the so-called ultra-faint $\mathrm{dSph}$ galaxies, which have very low masses, show a propensity to reach higher $[\mathrm{Mg} / \mathrm{Ca}]$ ratios as a result of a stochastical sampling of the high-mass end of the IMF, which in turn causes an imbalance between the $\mathrm{Mg}$ - and Ca-production (e.g., Koch et al. 2008a; Feltzing et al. 2009; Norris et al. 2010). In addition, the $\mathrm{dSph}$ galaxies show a clear extension towards low $[\mathrm{Mg} / \mathrm{Ca}]$ ratios, which reflects in an overall skewness of -0.13 in the $\mathrm{dSph}$ distribution. Notably, all of the "reference GCs" considered here (Table 5) have positive $\mathrm{Mg} / \mathrm{Ca}$ values. Given the rather large formal uncertainty of $\pm 0.30 \mathrm{dex}$ on the $[\mathrm{Mg} / \mathrm{Ca}] \mathrm{ra}-$ tio (adding the total errors on $\mathrm{Mg}$ and $\mathrm{Ca} / \mathrm{Fe}$ in quadrature) our measurement does not serve as an especially strong discriminator between halo field or dSph origin for Pal 4. Nevertheless, its value is clearly different from those of the remainder of inner and outer halo GCs, and may point to different enrichment processes in the environment where Pal 4 formed.

\subsection{Iron peak elements}

Our measured $[\mathrm{Sc} / \mathrm{Fe}],[\mathrm{Mn} / \mathrm{Fe}]$ and $[\mathrm{Ni} / \mathrm{Fe}]$ ratios are shown in Fig. 7. Owing to the relatively large number of available $\mathrm{Ni}$ absorption lines, $[\mathrm{Ni} / \mathrm{Fe}]$ is the best determined of these ratios,
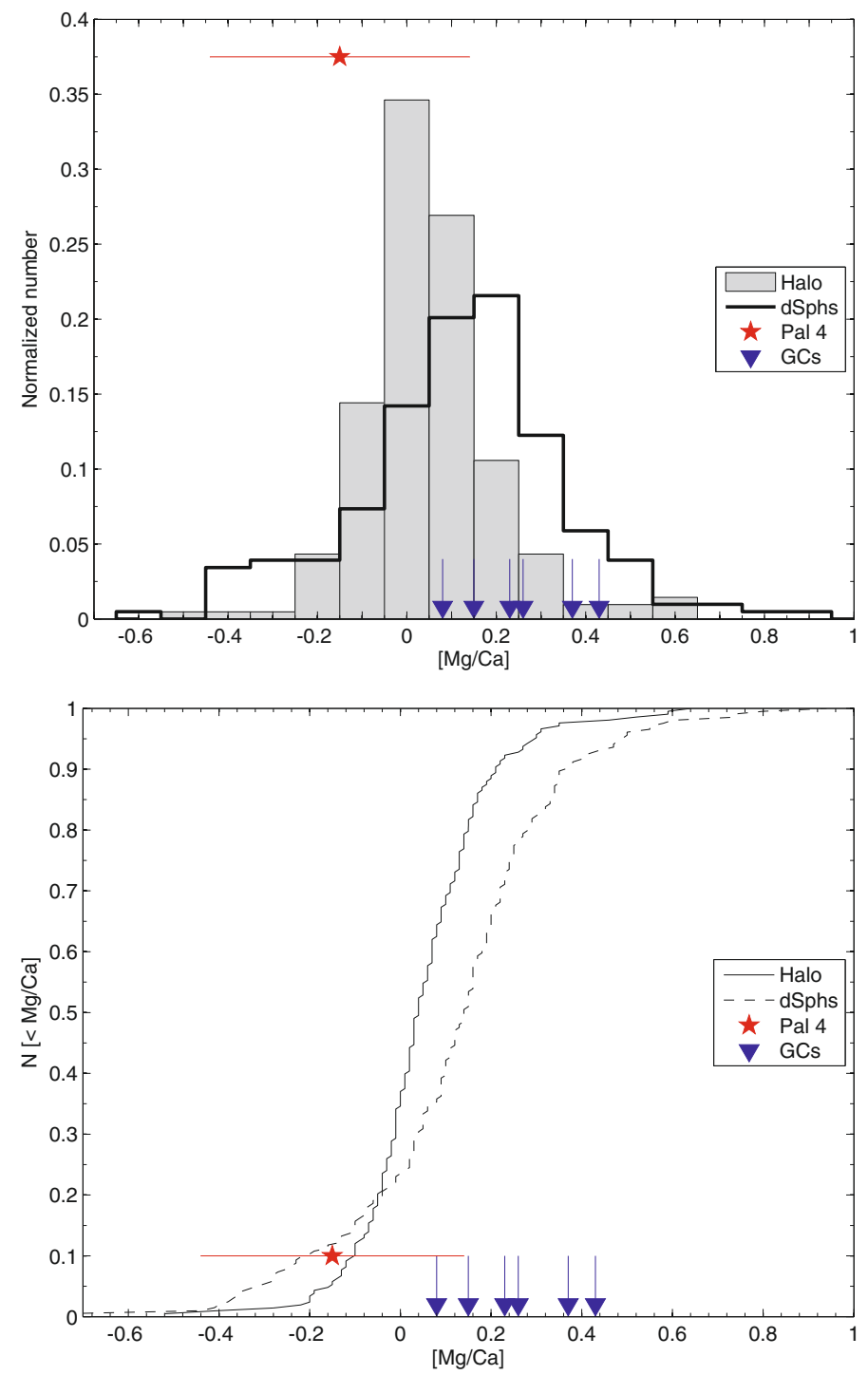

Fig. 6. Histograms (top panel) and cumulative distribution (bottom panel) of the $[\mathrm{Mg} / \mathrm{Ca}]$ abundance ratio in Galactic halo stars (shaded histogram/black solid line) and dSph galaxies (open histogram/dashed line). Also indicated are the measurements for Pal 4 and the Galactic GCs listed in Table 5 (see Sect. 5.4). The error bar on the Pal 4 data point is the squared sum of the total $\mathrm{Mg}$ and $\mathrm{Ca} / \mathrm{Fe}$ errors.

and, at $0.04 \mathrm{dex}$, has a value that is fully compatible with the Solar value (that is found over a broad range of iron abundances). This is not unexpected, since the iron-peak elements strictly 


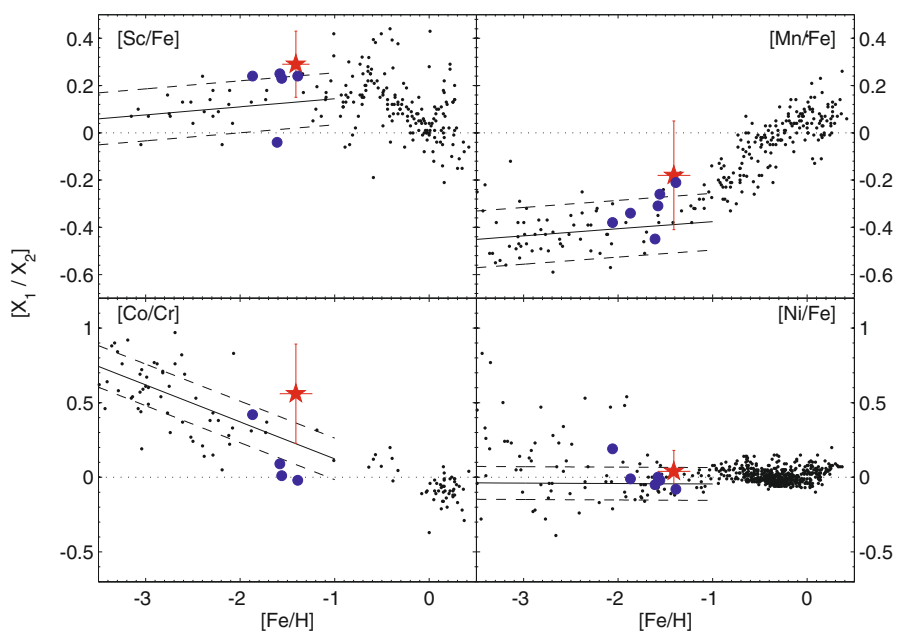

Fig. 7. Same as Fig. 5, but for $[\mathrm{Sc} / \mathrm{Fe}]$, $[\mathrm{Mn} / \mathrm{Fe}]$, $[\mathrm{Ni} / \mathrm{Fe}]$ and $[\mathrm{Co} / \mathrm{Cr}]$. Black lines denote the regression lines and $1 \sigma$ scatter adopted from Cayrel et al. (2004), extrapolated to $[\mathrm{Fe} / \mathrm{H}]=-1$ dex.

trace the iron production in the long-lived $\mathrm{SNe} \mathrm{Ia}$. $\mathrm{Cr}$ is underabundant with respect to $\mathrm{Fe}$, but fully compatible with Galactic halo stars, while $[\mathrm{Co} / \mathrm{Fe}]$ is slightly higher than halo stars at the same metallicity. In Fig. 7 , we choose to plot $[\mathrm{Co} / \mathrm{Cr}]$ as this abundance ratio has proven to be relatively insensitive to systematic effects in the stellar parameters (e.g., McWilliam et al. 1995). The high Co abundance in Pal 4, coupled with a relatively low $\mathrm{Cr}$ abundance, leads to the marginally higher $[\mathrm{Co} / \mathrm{Cr}]$ ratio indicated in this figure. Given its large uncertainty, and because we cannot rule out the possibility that this ratio has been affected by non-LTE effects, we will refrain from drawing any conclusions about the contributions from massive stars yields to these elements' production in Pal 4 (cf. McWilliam et al. 1995; Koch et al. 2008a).

Likewise, the $[\mathrm{Mn} / \mathrm{Fe}]$ ratio in $\mathrm{Pal} 4$, at $-0.18 \mathrm{dex}$, is marginally higher than the value of $\approx-0.4$ dex found for halo stars in the same $[\mathrm{Fe} / \mathrm{H}]$ interval (for which we supplemented the plot with data from Gratton 1989; Feltzing \& Gustafsson 1998; Prochaska et al. 2000; Nissen et al. 2000; Johnson 2002, and Cayrel et al. 2004; see also McWilliam et al. 2003). However, an intercomparison of Mn data usually suffers from zero point uncertainties (e.g., McWilliam et al. 2003) in that abundances derived from the $\sim 4030 \AA$ triplet lines are systematically lower by $0.3-0.4$ dex on average relative to the redder, high-excitation lines we employed in this study (e.g., Roederer et al. 2010). Thus Pal 4's elevated $[\mathrm{Mn} / \mathrm{Fe}]$ does not appear unusual and we do not pursue this ratio any further. Finally, the $[\mathrm{Cu} / \mathrm{Fe}]$ ratio (shown in Fig. 8 on top of the measurements in Galactic disk and halo stars by Prochaska et al. 2000; and Mishenina et al. 2002) seem to agree well with the Galactic trend, suggestive of a common origin, although zero-point difficulties may also affect conclusions about the behavior of this element (e.g., McWilliam \& Smecker-Hane 2005), as was the case for Mn.

\subsection{Neutron capture elements}

We show in Fig. 8 the $[\mathrm{Y} / \mathrm{Fe}]$ and $[\mathrm{Ba} / \mathrm{Fe}]$ ratios as representatives of the heavy elements.

All the elements with $Z>38$ are markedly enhanced relative to Fe. Unfortunately, our spectra lack information about the $r$-process element $\mathrm{Eu}$, which prohibits any conclusions about

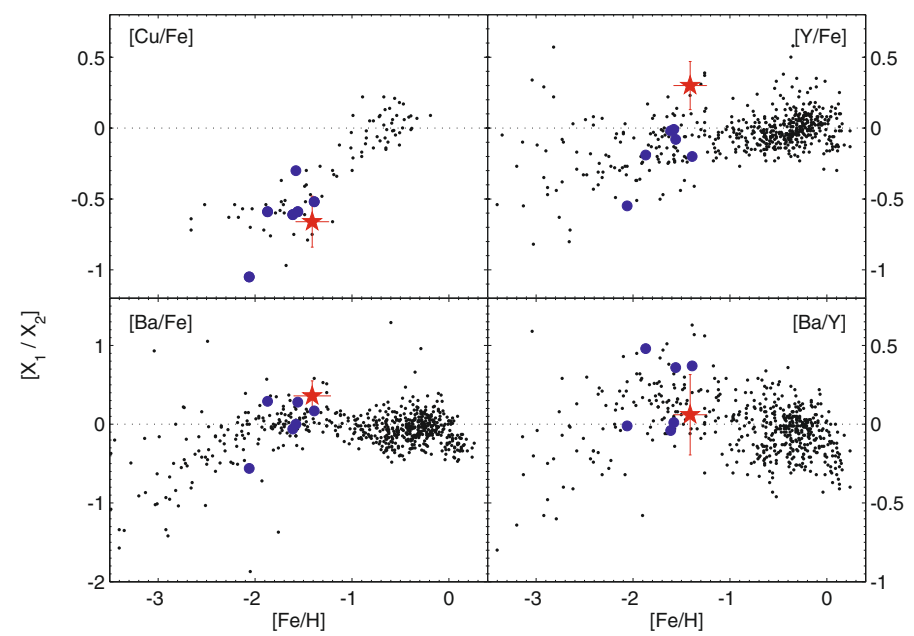

Fig. 8. Same as Fig. 7 , but for $[\mathrm{Cu} / \mathrm{Fe}],[\mathrm{Y} / \mathrm{Fe}],[\mathrm{Ba} / \mathrm{Fe}]$ and the $s$-process abundance ratio $[\mathrm{Ba} / \mathrm{Y}]$.

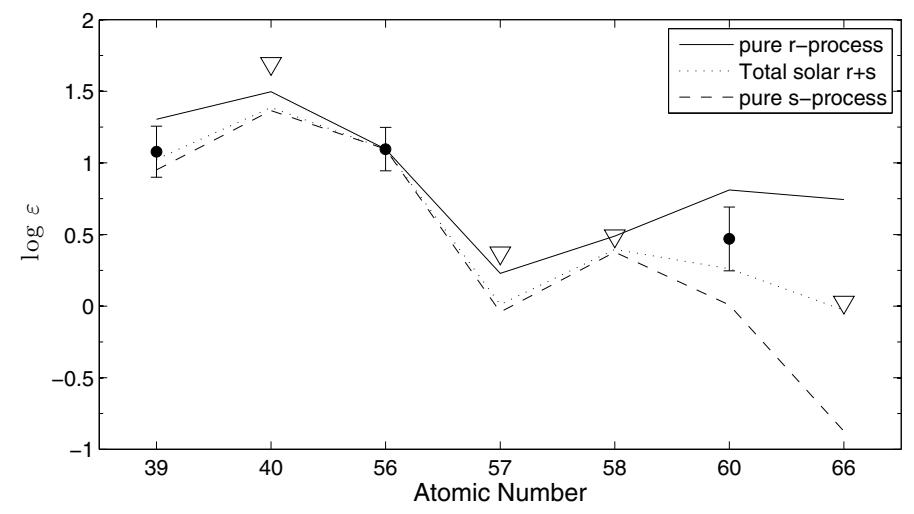

Fig. 9. Neutron capture elements in Pal 4, normalized to Ba. Black lines display the solar $r$ - and $s$-process contributions from Burris et al. (2000). Triangles indicate upper limits.

the relative contributions of the AGB stars that produce the $s$-process elements to the early $r$-process production (most likely in massive SNe II). On the other hand, the $[\mathrm{Ba} / \mathrm{Y}]$ of $\sim 0.06$ is fully compatible with the values found in Galactic halo stars, while it is strongly enhanced in the majority of the dSph stars studied to date owing to the importance of metal-poor AGB yields in the slow chemical evolution in these low-mass systems (e.g., Shetrone et al. 2003; Lanfranchi et al. 2008).

Figure 9 shows the heavy element abundances for Pal 4 together with the solar $r$-, $s$ - and total scaled solar abundances from Burris et al. (2000). We have normalized the curves to the same Ba abundance. Unlike Pal 3, which was found to exhibit interesting evidence for a pure $r$-process origin, Pal 4's abundance data fall between the $r$-process curve and the solar $r+s$-mix. However, the majority of these elements provide upper limits at most, so we refrain from a deeper discussion of the heavy element nucleosynthesis in this GC.

\section{Comparison to Galactic halo tracers}

\subsection{Halo globular clusters}

Figure 10 and Table 5 compare our abundances for Pal 4 to those of a subsample of Galactic GCs using data taken from the 


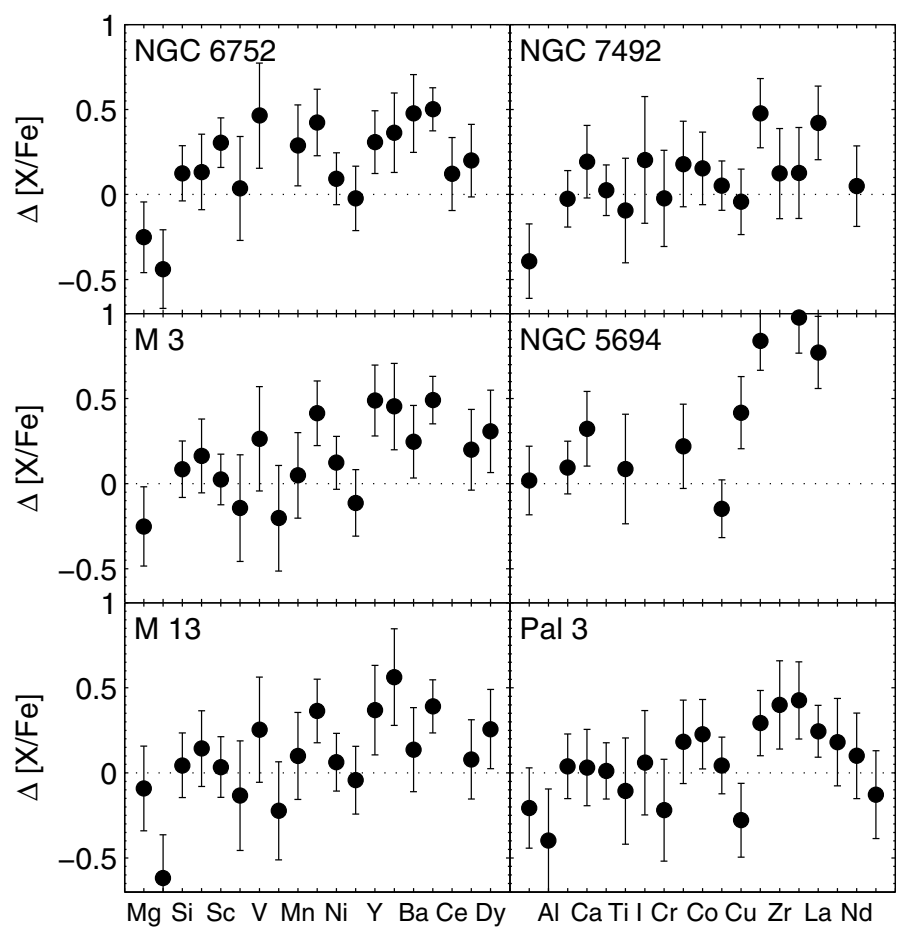

Fig. 10. Abundance differences for $\mathrm{Pal} 4$, in the sense $[\mathrm{X} / \mathrm{Fe}]_{\mathrm{Pal} 4}-$ $[\mathrm{X} / \mathrm{Fe}]_{\mathrm{GC}}$. The six GCs are results assembled from the literature and corrected for different Solar abundance scales. Details for the GCs used in this comparison are given in Table 5. Error bars include the $1 \sigma$-spreads from both this work and from the reference $\mathrm{GC}$ abundance ratios. For clarity, alternating labels are shown.

literature. Here we do not aim for a comprehensive comparison with the entire MWGC population (e.g., Pritzl et al. 2005; Geisler et al. 2007). Rather, we wish to simply compare Pal 4 to a few clusters that have been selected as broadly representative of the inner and outer halo cluster systems. Specifically, we use data for M 3 and M 13 from Cohen \& Meléndez (2005b), which are archetypical inner halo GCs at $R_{\mathrm{GC}} \sim 9,12 \mathrm{kpc}\left(Z_{\max } \sim 9\right.$, $15 \mathrm{kpc}$ ) with metallicities similar to those of Pal 4 . We also include NGC 6752 in this comparison as one of the nearest, inner halo clusters at a comparable metallicity (Yong et al. 2005). Finally, we include the outer halo clusters NGC 7492 (Cohen \& Meléndez 2005a) and Pal 3 (Paper I) as rare examples of remote clusters with published abundances, as well as NGC 5694, a GC that has been claimed to show abundance patterns more typical of dSph stars than GCs (Lee et al. 2006).

Table 5 shows the mean deviation $\langle\Delta[\mathrm{X} / \mathrm{Fe}]\rangle$ of Pal 4 's abundance ratios from the literature values for the GCs chosen for reference. The fourth column lists the number of chemical elements, $N$, that the different studies have in common. Since Figs. 8 and 9 indicate that Pal 4 exhibits relatively high heavy element ratios with regard to the reference sample, we also computed the statistics for elements with $Z<39$ (Y) only. This comparison suggests that $\mathrm{Pal} 4$ is, on average, enhanced with respect to each GC considered here if we account for all elements. On the other hand, the differences are statistically insignificant if we restrict the comparison to elements lighter than Y.

Two of the comparison GCs in Table 5 show interesting discrepancies. The first, NGC 6752, is the innermost object in the comparison sample and only slightly more metal-poor than Pal 4. Although its abundance patterns are similar to the comparison GCs and field stars at this metallicity, Yong et al. (2005, and references therein) found significant variations in the light and heavy elements, which supports the view that AGB stars alone cannot have carried the enrichment in the proto-cluster medium, although they likely played a significant role as indicated by the observed $[\mathrm{Ba} / \mathrm{Eu}]$ ratios. While the observed differences for $Z \gtrsim 39$ would seem to suggest that the respective processes differed between the inner (NGC 6752-like) and outer (Pal 4-like) halo, it is clear that more measurements - particularly Eu abundances - are needed.

The second noteworthy example, NGC 5694, exhibits heavy element abundance ratios that are incompatible with those of $\mathrm{Pal}$ 4. On the other hand, while its $[\mathrm{Ca} / \mathrm{Fe}]$ is also significantly lower, we find an identical $[\mathrm{Mg} / \mathrm{Fe}]$ ratio in $\mathrm{Pal} 4$ (which, in turn, reflects in the different $[\mathrm{Mg} / \mathrm{Ca}]$ ratios; see Fig. 6). The low values of the $\alpha$-element ratios with respect to the Galactic halo have prompted Lee et al. (2006) to conclude that this GC is likely of an extragalactic origin. We shall return to this issue below.

\subsection{Halo field stars}

Is it safe to conclude that Pal 4 is typical of the Galactic halo population? In view of the relatively large errors that arise from the integrated nature of our analysis, we follow Norris et al. (2010) in first considering the mean halo abundance distribution. To this end, we computed the mean and dispersion for the Galactic halo and disk stars (shown as small black dots in Figs. 5, 7 and 8) as a function of $[\mathrm{Fe} / \mathrm{H}]$ and fitted these relations with straight lines. Although this is an obvious oversimplification, the halo data is adequately represented with these linear relations. The resulting range in the Galactic abundance ratios is shown by the black lines in Fig. 5. We emphasize that no efforts have been taken to homogenize the various data with respect to different approaches used in the analyses (i.e., regarding $\log g f$ values and atmospheres), although we did correct for differences in the adopted Solar abundance scales when necessary. Note that Cayrel et al. (2004) also provide regression lines for [X/Fe] versus $[\mathrm{Fe} / \mathrm{H}]$ based on their 35 metal-poor halo stars, but those stars have $[\mathrm{Fe} / \mathrm{H}]<-2.1 \mathrm{dex}$ and an extrapolation to metallicities of Pal 4 yields slopes that are too high to describe the $\alpha$-element abundances shown here.

Pal 4 falls squarely on the regression lines for all $\alpha$-elements, except for $\mathrm{Ca}$, although it is still consistent within the errors even in this case. Indeed, Pal 4 is generally in good agreement with the GCs shown in this comparison, with the exceptions noted above. In this picture, the proto-GC cloud from which $\mathrm{Pal} 4$ formed was considerably enriched by the short-lived SNe II that produced the $\alpha$-elements on rapid time scales - a generic characteristic of the halo field stars and its genuine GC system.

For the even- and odd-Z iron-peak element ratios shown in Fig. 7, an extrapolation of the regression lines of Cayrel et al. (2004) provide good representations of the overall halo trends up to the metallicity regime around Pal 4 and higher. As argued above, the $[\mathrm{Ni} / \mathrm{Fe}]$ ratio is well determined in Pal 4 and is fully compatible with the Solar value that is observed in halo field and GC stars, bolstering the ubiquity of iron-peak nucleosynthesis in the $\mathrm{SNe} \mathrm{Ia}$ at $[\mathrm{Fe} / \mathrm{H}]$ above $\sim-2$ dex. The $[\mathrm{Sc} / \mathrm{Fe}]$ ratio in Pal 4 falls towards the upper limit of the halo distribution, which holds for all the reference GCs in our sample, except NGC 6752. Likewise, the slight Mn enhancement is not atypical and agrees well with, for instance, M 3. This may indicate that the metallicity dependence of the SNe II yields was less pronounced in the Pal 4 proto-GC cloud (cf. McWilliam et al. 2003). Finally, we note that the $[\mathrm{Co} / \mathrm{Cr}]$ ratio is significantly larger that those observed for the three GCs in this metallicity 
range. These GCs show roughly solar values, as would be expected as both elements fall close together on the iron peak. McWilliam et al. (1995) first detected a strong rise of this ratio in metal-poor halo stars below $\sim-2.4$ dex. In fact, the observed $[\mathrm{Co} / \mathrm{Cr}]$ of $0.55 \pm 0.33 \mathrm{dex}$ is reminiscent of NGC 7492, albeit at a metallicity that is higher by roughly 0.5 dex.

In the case of $\mathrm{Cu}$, and the $n$-capture elements $\mathrm{Y}$ and $\mathrm{Ba}$, the scatter in the halo abundance ratios is more difficult to evaluate due to a much sparser sampling of those elements and a notably increased (and real) abundance scatter among the metalpoor stars below $\sim-2$ dex. We therefore restrict the following brief discussion of Fig. 8 to the scatter plots without quantifying any linear trends.

While the $[\mathrm{Y} / \mathrm{Fe}]$ ratio lies above the bulk of the halo data, and is also higher than our comparison clusters by more than 0.3 dex, Ba seems only mildy enhanced with respect to these populations. Overall, the $s$-process ratio $[\mathrm{Ba} / \mathrm{Y}]$ is in full agreement with the halo fields stars within the scatter. However, Pritzl et al. (2005) have shown that, in comparison with (thick) disk $\mathrm{GCs}$, the halo clusters tend to be offset more towards higher $[\mathrm{Ba} / \mathrm{Y}]$ ratios, and so are the dSphs. The latter is usually interpreted in terms of the low star formation efficiencies of the $\mathrm{dSph}$ galaxies, which leaves room for a much stronger contribution from metal-poor AGB stars that are the main sites of the $s$-process (e.g., Busso et al. 2001; Lanfranchi et al. 2008). The three $\mathrm{GCs}$ with the very high $[\mathrm{Ba} / \mathrm{Y}]$ ratios in Fig. 10 are M 3, M 13 and NGC 7492 and therefore representatives of the inner and outer halo. Following this line of reasoning, the slow star forming rates and metallicity dependent AGB-yields that cause enhancements in this ratio appear to be unrelated to location within the halo. In Paper I we found that Pal 3's heavy elements are largely governed by $r$-process nucleosynthesis. From the sparse data for $Z>38$ in NGC 5496, it cannot be excluded that this cluster also follows this trend, so that the above arguments regarding bimodal $s$-process ratios may not apply to these remote halo clusters. In any case, we emphasize that detailed $r$ and $s$-process abundance measurements for individual stars are vital for resolving these questions.

\subsection{Comparison with other substructures in the outer halo}

In this section, we compare our abundances for Pal 3 (Paper I) and Pal 4 to published values for other "substructures" or "overdensities" in the outer halo of the Milky Way, regardless of their morphological classification. Our comparison therefore focuses on a sample of 13 halo GCs, seven dSph galaxies (Sagittarius, Fornax, Draco, Sextans, Carina, Ursa Minor and Leo II) with abundance data from Shetrone et al. (2001, 2003, 2009); Sadakane et al. (2004); Monaco et al. (2005); Letarte (2007); Koch et al. (2008b); Cohen \& Huang (2009); Aoki et al. (2009); and the five so-called "ultra-faint" dSph galaxies (hereafter UF-dSphs; Hercules, Coma Berenices, Ursa Major II, Bootes I and Leo IV) with published abundance information (Koch et al. 2008a; Frebel et al. 2010; Feltzing et al. 2009; Simon et al. 2010).

All GCs shown here were selected to have Galactocentric distances $R_{\mathrm{GC}} \gtrsim 8 \mathrm{kpc}$; including Pal 3 and $\mathrm{Pal} 4$ gives us a total of five GCs beyond $R_{\mathrm{GC}}=25 \mathrm{kpc}$, and three of these GCs (Pal 3, Pal 4 and NGC 2419) are at $R_{\mathrm{GC}} \geq 90 \mathrm{kpc}$. Note that only two other GCs in the catalog of Harris (1996) lie at or beyond this distance (Eridanus and AM 1). Thus, while the available abundance measurements are certainly still sparse (i.e., being based on just a single RGB star in NGC 2419, four RGB stars in Pal 3, and co-added spectra for 19 RGB stars in Pal 4;
Shetrone et al. 2001; Paper I), it is now possible to have first glimpse into the abundance patterns of the most remote Galactic GCs, and their relationship, if any, to the dSph and UF-dSph galaxies residing in the outer halo. Because the number of element abundance measurements is generally limited (and differs amongst the various studies), we restrict our comparison to $[\mathrm{Fe} / \mathrm{H}]$ and $[\alpha / \mathrm{Fe}]$, where we take $[\alpha / \mathrm{Fe}] \equiv([\mathrm{Mg} / \mathrm{Fe}]+[\mathrm{Ca} / \mathrm{Fe}]$ $+[\mathrm{Ti} / \mathrm{Fe}]) / 3$.

In Fig. 11, we show the behaviour of $[\mathrm{Fe} / \mathrm{H}]$ and $[\alpha / \mathrm{Fe}]$ for stars belonging to halo GCs (blue squares), the more luminous dSph galaxies (orange circles) and UF-dSph galaxies (brown circles). Note that we plot GCs in the range $8 \geq R_{\mathrm{GC}} \geq$ $25 \mathrm{kpc}$ as open blue squares, while GCs in the range $R_{\mathrm{GC}} \geq$ $25 \mathrm{kpc}$ are shown as filled blue squares. Abundances are plotted against total $V$-band magnitude, $M_{V}$, central $V$-band surface brightness, $\mu_{V}(0)$, and effective (or half-light) radius, $R_{\mathrm{e}}$ (Harris 1996; Irwin \& Hatzidimitriou 1995; Mateo 1998; McLaughlin \& van der Marel 2005; Martin et al. 2008). Pal 3 and 4 are highlighted as the large red square and star, respectively, while the third GC at $R_{\mathrm{GC}} \gtrsim 90 \mathrm{kpc}$, NGC 2419 , is labelled in each panel.

There are several interesting conclusions to be drawn from this figure. First, Pal 3 and 4 appear as near "twins" in this comparison, having similar Galactocentric distances, structural parameters (notably large radii), $V$-band luminosities, metallicities and $\alpha$-element enhancements. NGC 2419, although much more luminous than either Pal 3 or Pal 4, appears similar in terms of its $\alpha$-enhancement. For these three GCs, which lie in the range $91 \lesssim R_{\mathrm{GC}} \lesssim 112 \mathrm{kpc}$, we find a mean of

$\langle[\alpha / \mathrm{Fe}]\rangle=+0.31 \pm 0.09$ dex.

Adding NGC 5694 and NGC 7006, we find

$\langle[\alpha / \mathrm{Fe}]\rangle=+0.24 \pm 0.13 \operatorname{dex}$

for the five GCs with $R_{\mathrm{GC}} \gtrsim 25 \mathrm{kpc}$. Thus, on the whole, Pal 3 and Pal 4 seem to have levels of $\alpha$ enhancement that are similar to most other halo GCs and nearby halo field stars, but slightly higher than dSphs at comparable metallicities (e.g., Shetrone et al. 2001, 2003; Venn et al. 2004; Koch 2009). It is important to bear in mind, however, that stars in individual dSph galaxies show significant scatter, and it is certainly true that some dSph stars fall close to the region in the $[\mathrm{Fe} / \mathrm{H}]-[\alpha / \mathrm{Fe}]$ diagram occupied by these remote GCs: i.e., $10 / 157 \approx 6 \%$ of the $\mathrm{dSph}$ stars plotted in Fig. 11 fall within the $2 \sigma$ uncertainties for Pal 3 and Pal 4.

In absolute terms, the mean $[\alpha / \mathrm{Fe}]$ for the most remote GCs is indistinguishable from that found in the UF-dSph galaxies shown in Fig. 11, which have $+0.36 \pm 0.17$ dex and a full range of +0.03 to +0.65 dex (based on measurements for nine stars in Her, UMa II, Com, and Leo IV). Note that Pal 3 and 4 are atypical of Galactic GCs in terms of their structural parameters, being unusually extended $\left(R_{\mathrm{e}} \gtrsim 15 \mathrm{pc}\right.$, or roughly fives times as large as "typical" GCs; Jordán et al. 2005) and having low surface brightness (with $\mu_{V}(0) \gtrsim 22-22.5 \mathrm{mag} \operatorname{arcsec}^{-2}$ ). Thus, at least superficially, these remote GCs may have more in common with some UF-dSph galaxies than their apparent counterparts in the inner halo.

There are, at present, two characteristics of the UF-dSph population that suggest they are indeed low-luminosity galaxies rather than faint, extended GCs (e.g., Larsen \& Brodie 2002; Mackey et al. 2006; Peng et al. 2006). The first such characteristic is their very large mass-to-light ratios, which point to the presence of significant dark matter halos (Simon \& Geha 2007; Strigari et al. 2008). Secondly, the UF-dSphs seem to have 


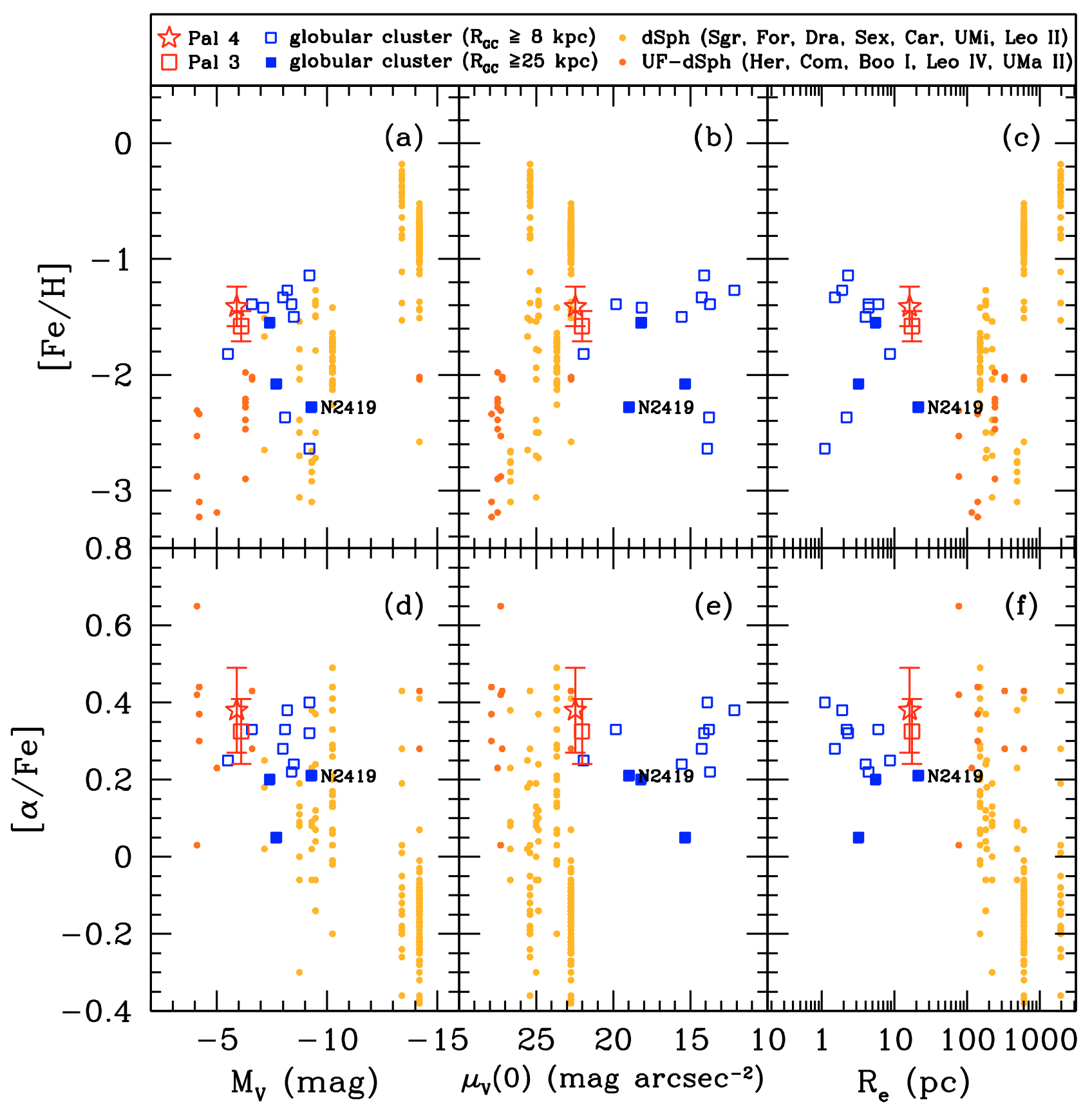

Fig. 11. Dependence of $[\mathrm{Fe} / \mathrm{H}]$ and $[\alpha / \mathrm{Fe}]$ on structural parameters for various types overdensities in the Galactic halo: globular clusters (open and filled blue squares), dSph galaxies (orange circles) and "ultra-faint" dSph galaxies (brown circles). The large red symbols show our results for Pal 3 (Paper I) and Pal 4 (this paper). The luminous globular cluster NGC 2419, which lies at a Galactocentric distance comparable to Pal 3 and $\mathrm{Pal}$ 4, is labeled in each panel. The structural parameters shown in this figure are absolute $V$-band magnitude (panels a,d), central $V$-band surface brightness (panels $\mathbf{b}, \mathbf{e}$ ) and effective radius (panels $\mathbf{c}, \mathbf{f}$ ).

abundances that fall along the extrapolation of the dwarf galaxy metallicity-luminosity relation, with significant intrinsic dispersions in metallicity (Kirby et al. 2008). Using these criteria, what can we conclude about the origin of the most remote halo GCs?

Unfortunately, it is difficult to draw firm conclusions on possible metallicity spreads in these systems since there are measurements for just a single RGB star in NGC 2419 (Shetrone et al. 2001), and our analysis of co-added spectra in Pal 4 presupposes that there is no abundance spread (see Sects. 1, 5.2). In the case of Pal 3, where high-quality MIKE spectra are available for four RGB stars, we can confidently rule out an abundance spread larger than $\sim 0.1$ dex (Paper I). Regarding the dark matter content of these systems, Baumgardt et al. (2009) have recently carried out a dynamical analysis of NGC 2419, finding $M / L_{V}=2.05 \pm 0.50$ in solar units. This value is typical of GCs (McLaughlin \& van der Marel 2005) and much smaller than the extreme values reported for UF-dSphs (e.g., Simon \& Geha 2007; Strigari et al. 2008). Detailed dynamical modeling of Pal 3 and 4 will be the subject of a future paper in this series, but it is clear that the extreme $M / L_{V}$ values for UF-dSph galaxies can be ruled out at a very high confidence (i.e., for a system like Pal 4, with $L_{V} \sim 2.1 \times 10^{4} L_{V, \odot}$, known UF-dSphs have mass-to-light ratios of $\approx 10^{3}$ to $10^{4}$; Strigari et al. 2008; Geha et al. 2009). 
In short, the available evidence suggests that Pal 4 (and Pal 3) formed in a manner resembling that of typical halo GCs, although it is clear that additional abundance measurements for stars in these and other remote GCs is needed urgently. Indeed, each contains many RGB stars that are well within the reach of high-resolution spectrographs on $8 \mathrm{~m}$-class telescopes. Such observations would allow a direct measurement of the intrinsic abundance spread within these systems - an important clue to their origin and relationship to other halo substructures such as $\mathrm{dSph}$ and UF-dSph galaxies.

\section{Summary}

Motivated by the good agreement between the abundance ratios measured from high- $S / N$ spectra of individual stars in Pal 3 and those found using co-added, low- $S / N$ spectra (Paper I), we have used the same technique to measure chemical abundance ratios in the remote halo GC Pal 4. Although systematic uncertainties and the low $S / N$ ratios complicate such studies, an accuracy of 0.2 dex is possible for most abundance ratios, sufficient to place such faint and remote systems into a context with both the inner and outer halo GCs, as well as dSph and UF-dSph galaxies. In the future, this technique may enable the global abundance patterns to be characterized in additional remote systems, allowing a first reconnaissance of the chemical enrichment histories of remote Galactic satellites.

Perhaps the most striking finding in Pal 4 is the subsolar $[\mathrm{Mg} / \mathrm{Ca}]$ ratio, which is not observed in the sample of reference GCs that span a broad range of Galactocentric distances. Despite an overlap of our observed ratio with the halo field population, its low value may rather resemble the low- $[\mathrm{Mg} / \mathrm{Ca}]$ tail of the distribution for $\mathrm{dSph}$ stars. In contrast, we see tentative evidence for a solar $[\mathrm{Ba} / \mathrm{Y}]$ ratio, which militates against a slow chemical evolution and accompanying AGB enrichment as suggested by enhanced $[\mathrm{Ba} / \mathrm{Y}]$ values in about two thirds of the dSph stars studied to date. Overall, most of the element ratios determined in this study overlap with the corresponding measurements for halo field stars, although a few ratios seem to fall above the halo star trends (see Sect. 5). This favors a scenario in which the material from which both Pal 4 and the Galactic halo formed underwent rather similar enrichment processes.

In their analysis of the CMD of Pal 4, Stetson et al. (1999) state that the cluster is younger than the inner halo GC M5 by about $1.5 \mathrm{Gyr}($ at $[\mathrm{Fe} / \mathrm{H}]=-1.33 \mathrm{dex}$; Ivans et al. 2003; Koch \& McWilliam 2010) if they "all have the same composition - and $[\ldots]$ this means both $[\mathrm{Fe} / \mathrm{H}]$ and $[\alpha / \mathrm{Fe}]$ ". Our work has shown that Pal 4 is enhanced by $+0.38 \pm 0.11$ dex in the $\alpha$-elements, which is consistent with the value of 0.3 dex assumed in the above CMD modeling. On the other hand, the CMD analysis suggested an $[\mathrm{Fe} / \mathrm{H}]$ of $-1.28 \mathrm{dex}$, which is slightly more metal rich than what we found in the present spectroscopic study: $\langle[\mathrm{Fe} / \mathrm{H}]\rangle=-1.41$ dex. As noted in Vandenberg (2000), "an increase in $[\mathrm{Fe} / \mathrm{H}]$ or $[\alpha / \mathrm{Fe}]$ would result in slightly younger $[\ldots]$ ages" for Pal 4 (as determined via the magnitude offset between the horizontal branch and main-sequence turnoff). This would imply that Pal 4 is slightly older than found in Stetson et al. (1999) and hence more similar in age to the older halo population. This, however, is in contradiction to the younger age suggested by its peculiar (i.e., red) horizontal branch morphology, unless further parameters, such as red giant mass loss, are invoked (Catelan 2000).

Based on the evidence at hand, Pal 4 seems to have an abundance pattern that is typical of other remote GCs in the outer halo. An open question, given the nature of our analysis which relies on co-adding individual RGB star spectra, is whether Pal 4 is monometallic or, like dSph and UF-dSph galaxies, shows an internal spread in metallicity. We argued in Sect. 5.2. judging from our limited quality spectra, however, that it is unlikely that this object exhibits any significant intrinsic iron scatter. It is clear that high-quality abundance ratio measurements for individual stars in Pal 4 and other remote substructures are urgently needed to understand the relationship, if any, between remote GCs and other substructures in the outer halo.

Acknowledgements. We thank I. U. Roederer for discussions and an anonymous referee for a very helpful report. A.K. acknowledges support by an STFC postdoctoral fellowship. This work was based on observations obtained at the W. M. Keck Observatory, which is operated jointly by the California Institute of Technology and the University of California. We are grateful to the W. M. Keck Foundation for their vision and generosity. We recognize the great importance of Mauna Kea to both the native Hawaiian and astronomical communities, and we are grateful for the opportunity to observe from this special place.

\section{References}

Alonso, A., Arribas, S., \& Martínez-Roger, C. 1999, A\&AS, 140, 261 Aoki, W., Arimoto, N., Sadakane, K., et al. 2009, A\&A, 502, 569 Armandroff, T. E., Da Costa, G. S., \& Zinn, R. 1992, AJ, 104, 164 Asplund, M., Grevesse, N., Sauval, A. J., \& Scott, P. 2009, ARA\&A, 47, 481 Baumgardt, H., Côté, P., Hilker, M., et al. 2009, MNRAS, 396, 2051 Belokurov, V., Zucker, D. B., Evans, N. W., et al. 2007, ApJ, 654, 897 Brodie, J. P., \& Larsen, S. S. 2002, AJ, 124, 1410

Bullock, J. S., Kravtsov, A. V., \& Weinberg, D. H. 2001, ApJ, 548, 33 Burris, D. L., Pilachowski, C. A., Armandroff, T. E., et al. 2000, ApJ, 544, 302 Busso, M., Gallino, R., Lambert, D. L., Travaglio, C., \& Smith, V. V. 2001, ApJ, 557,802

Carretta, E., \& Gratton, R. 1997, A\&AS, 121, 95

Carretta, E., Bragaglia, A., Gratton, R., D’Orazi, V., \& Lucatello, S. 2009, A\&A, 508,695

Castelli, F., \& Kurucz, R. L. 2003, Modelling of Stellar Atmospheres, 210, 20P Catelan, M. 2000, ApJ, 531, 826

Cayrel, R., Depagne, E., Spite, M., et al. 2004, A\&A, 416, 1117

Cohen, J. G., \& Huang, W. 2009, ApJ, 701, 1053

Cohen, J. G., \& Melendez, J. 2005a, AJ, 129, 1607

Cohen, J. G., \& Meléndez, J. 2005b, AJ, 129, 303

Côté, P., Djorgovski, S. G., Meylan, G., Castro, S., \& McCarthy, J. K. 2002, ApJ, 574,783

Dotter, A., Chaboyer, B., Jevremović, D., et al. 2008, ApJS, 178, 89

Dotter, A., Sarajedini, A., Anderson, J., et al. 2010, ApJ, 708, 698

Feltzing, S., \& Gustafsson, B. 1998, A\&AS, 129, 237

Feltzing, S., Eriksson, K., Kleyna, J., \& Wilkinson, M. I. 2009, A\&A, 508, L1

Font, A. S., Johnston, K. V., Bullock, J. S., \& Robertson, B. E. 2006, ApJ, 638, 585

Frebel, A., Simon, J. D., Geha, M., \& Willman, B. 2010, ApJ, 708, 560

Fulbright, J. P. 2000, AJ, 120, 1841

Fulbright, J. P. 2002, AJ, 123, 404

Geha, M., Willman, B., Simon, J. D., et al. 2009, ApJ, 692, 1464

Geisler, D., Wallerstein, G., Smith, V. V, \& Casetti-Dinescu, D. I. 2007, PASP, 119,939

Gratton, R. G. 1989, A\&A, 208, 171

Gratton, R. G., \& Sneden, C. 1988, A\&A, 204, 193

Gratton, R. G., \& Sneden, C. 1994, A\&A, 287, 927

Hanson, R. B., Sneden, C., Kraft, R. P., \& Fulbright, J. 1998, AJ, 116, 1286

Harris, W. E. 1996, AJ, 112, 1487

Irwin, M., \& Hatzidimitriou, D. 1995, MNRAS, 277, 1354

Ivans, I. I., Sneden, C., James, C. R., et al. 2003, ApJ, 592, 906

Johnson, J. A. 2002, ApJS, 139, 219

Jordán, A., Côté, P., Blakeslee, J. P., et al. 2005, ApJ, 634, 1002

Jordi, K., Grebel, E. K., Hilker, M., et al. 2009, AJ, 137, 4586

Kirby, E. N., Simon, J. D., Geha, M., Guhathakurta, P., \& Frebel, A. 2008, ApJ, $685, \mathrm{~L} 43$

Klypin, A., Kravtsov, A. V., Valenzuela, O., \& Prada, F. 1999, ApJ, 522, 82

Koch, A. 2009, Astron. Nachr., 330, 675

Koch, A., \& McWilliam, A. 2010, AJ, 139, 2289

Koch, A., McWilliam, A., Grebel, E. K., Zucker, D. B., \& Belokurov, V. 2008a, ApJ, 688, L13

Koch, A., Grebel, E. K., Gilmore, G. F., et al. 2008b, AJ, 135, 1580

Koch, A., Rich, R. M., Reitzel, D. B., et al. 2008c, ApJ, 689, 958

Koch, A., Côté, P., \& McWilliam, A. 2009, A\&A, 506, 729 (Paper I)

Kraft, R. P., \& Ivans, I. I. 2003, PASP, 115, 143

Lai, D. K., Rockosi, C. M., Bolte, M., et al. 2009, ApJ, 697, L63 
Lanfranchi, G. A., \& Matteucci, F. 2004, MNRAS, 351, 1338

Lanfranchi, G. A., Matteucci, F., \& Cescutti, G. 2008, A\&A, 481, 635

Larsen, S. S., \& Brodie, J. P. 2002, AJ, 123, 1488

Lee, J.-W., López-Morales, M., \& Carney, B. W. 2006, ApJ, 646, L119

Lee, J.-W., Kang, Y.-W., Lee, J., \& Lee, Y.-W. 2009, Nature, 462, 480

Letarte, B. 2007, Ph.D. Thesis, University of Groningen

Li, Y.-S., De Lucia, G., \& Helmi, A. 2009, MNRAS, 1727

Mackey, A. D., \& Gilmore, G. F. 2004, MNRAS, 355, 504

Mackey, A. D., Huxor, A., Ferguson, A. M. N., et al. 2006, ApJ, 653, L105

Martin, N. F., de Jong, J. T. A., \& Rix, H.-W. 2008, ApJ, 684, 1075

Mateo, M. L. 1998, ARA\&A, 36, 435

McLaughlin, D. E., \& van der Marel, R. P. 2005, ApJS, 161, 304

McWilliam, A. 1998, AJ, 115, 1640

McWilliam, A., \& Bernstein, R. A. 2008, ApJ, 684, 326

McWilliam, A., \& Smecker-Hane, T. A. 2005, ApJ, 622, L29

McWilliam, A., Preston, G. W., Sneden, C., \& Searle, L. 1995, AJ, 109, 2757

McWilliam, A., Rich, R. M., \& Smecker-Hane, T. A. 2003, ApJ, 592, L21

Mishenina, T. V., Kovtyukh, V. V., Soubiran, C., Travaglio, C., \& Busso, M. 2002, A\&A, 396, 189

Monaco, L., Bellazzini, M., Bonifacio, P., et al. 2005, A\&A, 441, 141

Nissen, P. E., \& Schuster, W. J. 1997, A\&A, 326, 751

Nissen, P. E., Chen, Y. Q., Schuster, W. J., \& Zhao, G. 2000, A\&A, 353, 722

Norris, J. E., Christlieb, N., Korn, A. J., et al. 2007, ApJ, 670, 774

Norris, J. E., Yong, D., Gilmore, G., \& Wyse, R. F. G. 2010, ApJ, 711, 350

Oke, J. B., Cohen, J. G., Carr, M., et al. 1995, PASP, 107, 375

Olszewski, E. W., Aaronson, M., \& Peterson, R. C. 1986, ApJ, 302, L45

Peng, E. W., Côté, P., Jordán, A., et al. 2006, ApJ, 639, 838

Prochaska, J. X., Naumov, S. O., Carney, B. W., McWilliam, A., \& Wolfe, A. M. 2000, AJ, 120, 2513
Pritzl, B. J., Venn, K. A., \& Irwin, M. 2005, AJ, 130, 2140

Ramírez, I., \& Meléndez, J. 2005, ApJ, 626, 465

Roederer, I. U., Sneden, C., Thompson, I. B., Preston, G. W., \& Shectman, S. A. 2010, ApJ, 711, 573

Ryan, S. G., Norris, J. E., \& Beers, T. C. 1996, ApJ, 471, 254

Sadakane, K., Arimoto, N., Ikuta, C., et al. 2004, PASJ, 56, 1041

Saha, A., Dolphin, A. E., Thim, F., \& Whitmore, B. 2005, PASP, 117, 37

Searle, L., \& Zinn, R. 1978, ApJ, 225, 357

Shetrone, M. D., Côté, P., \& Sargent, W. L. W. 2001, ApJ, 548, 592

Shetrone, M., Venn, K. A., Tolstoy, E., et al. 2003, AJ, 125, 684

Shetrone, M. D., Siegel, M. H., Cook, D. O., \& Bosler, T. 2009, AJ, 137, 62

Simon, J. D., \& Geha, M. 2007, ApJ, 670, 313

Simon, J. D., Frebel, A., McWilliam, A., Kirby, E. N., \& Thompson, I. B. 2010, ApJ, 716, 446

Skrutskie, M. F., Cutri, R. M., Stiening, R., et al. 2006, AJ, 131, 1163

Sneden, C. A. 1973, Ph.D. Thesis, The University of Texas at Austin

Stephens, A., \& Boesgaard, A. M. 2002, AJ, 123, 1647

Stetson, P. B., Bolte, M., Harris, W. E., et al. 1999, AJ, 117, 247

Strigari, L. E., Bullock, J. S., Kaplinghat, M., et al. 2008, Nature, 454, 1096

VandenBerg, D. A. 2000, ApJS, 129, 315

Venn, K. A., Irwin, M., Shetrone, M. D., et al. 2004, AJ, 128, 1177

Vogt, S. S., Allen, S. L., Bigelow, B. C., et al. 1994, Proc. SPIE, 2198, 362

Walker, M. G., Mateo, M., Olszewski, E. W., et al. 2007, ApJS, 171, 389

Winkler, H. 1997, MNRAS, 287, 481

Woosley, S. E., \& Weaver, T. A. 1995, ApJS, 101, 181

Yong, D., Grundahl, F., Nissen, P. E., Jensen, H. R., \& Lambert, D. L. 2005, A\&A, 438, 875

Zinn, R., \& West, M. J. 1984, ApJS, 55, 45

Pages 14 to 15 are available in the electronic edition of the journal at http://www . aanda.org 
Table 1. Observation log and properties of the target stars.

\begin{tabular}{|c|c|c|c|c|c|c|c|c|c|}
\hline $\mathrm{ID}^{a}$ & Date & $\begin{array}{c}\text { Exposure time } \\
{[\mathrm{s}]}\end{array}$ & $\begin{array}{c}\alpha \\
(\mathrm{J} 2000.0)\end{array}$ & $\begin{array}{c}\delta \\
(\mathrm{J} 2000.0)\end{array}$ & $\begin{array}{c}V \\
{[\mathrm{mag}]}\end{array}$ & $\begin{array}{l}B-V \\
{[\mathrm{mag}]}\end{array}$ & $\begin{array}{l}V-I \\
{[\mathrm{mag}]}\end{array}$ & $\begin{array}{l}V-K \\
{[\mathrm{mag}]}\end{array}$ & $\begin{array}{c}S / N \\
{\left[\text { pixel }^{-1} \text { ] }\right.}\end{array}$ \\
\hline Pal4-1 (S196) & Feb. 11, 1999, Mar. 10, 1999 & $3 \times 300$ & 112917.13 & +285759.9 & 17.81 & 1.46 & 1.52 & 3.43 & 8 \\
\hline Pal4-2 (S169) & Feb. 11, 1999 & $2 \times 300$ & 112917.02 & +285751.5 & 17.93 & 1.46 & 1.45 & 3.57 & 8 \\
\hline Pal4-3 (S277) & Feb. 11, 1999 & $1 \times 300$ & 112913.24 & +2858 13.6 & 17.82 & 1.66 & 1.53 & 3.69 & 8 \\
\hline Pal4-5 (S434) & Feb. 11, 1999, Feb. 12, 1999 & $2 \times 300$ & 112916.67 & +285842.1 & 17.95 & 1.44 & 1.47 & 2.81 & 7 \\
\hline Pal4-6 (S158) & Feb. 11, 1999, Mar. 10, 1999 & $3 \times 420$ & 112915.50 & +285747.0 & 18.22 & 1.30 & 1.33 & 3.06 & 7 \\
\hline Pal4-7 (S381) & Feb. 11, 1999, Mar. 10, 1999 & $2 \times 600$ & 112914.83 & +285832.2 & 18.55 & 1.19 & 1.24 & 2.79 & 7 \\
\hline Pal4-8 (S364) & Feb. 11,1999 & $1 \times 600$ & 112912.66 & +285829.6 & 18.65 & 1.17 & 1.23 & 2.85 & 6 \\
\hline Pal4-9 (S534) & Feb. 11, 1999 & $1 \times 750$ & 112913.32 & +285907.6 & 19.00 & 1.08 & 1.18 & 3.62 & 6 \\
\hline Pal4-10 (S325) & Feb. 11, 1999 & $2 \times 900$ & 112915.71 & +285723.4 & 19.09 & 1.05 & 1.13 & $\ldots$ & 6 \\
\hline Pal4-11 (S430) ${ }^{b}$ & Feb. 11, 1999 & $1 \times 1200$ & 112913.82 & +285840.9 & 19.35 & 0.89 & 1.04 & $\ldots$ & 5 \\
\hline Pal4-12 (S328) ${ }^{b}$ & Feb. 11, 1999, Mar. 10, 1999 & $1 \times 1200,1 \times 2400$ & 112917.63 & +285825.1 & 19.35 & 0.90 & 1.03 & $\ldots$ & 8 \\
\hline Pal4-15 (S307) $)^{b}$ & Feb. 11, 1999 & $1 \times 1200$ & 112916.45 & +285818.4 & 19.38 & 0.88 & 1.00 & $\ldots$ & 5 \\
\hline Pal4-16 (S306) ${ }^{b}$ & Feb. 11, 1999 & $1 \times 1200$ & 112917.77 & +285819.5 & 19.43 & 0.88 & 1.02 & $\ldots$ & 5 \\
\hline Pal4-17 (S472) ${ }^{b}$ & Feb. 12, 1999 & $1 \times 1080$ & 112915.95 & +285847.8 & 19.45 & 0.85 & 0.99 & & 5 \\
\hline Pal4-18 (S186) & Feb. 11, 1999 & $1 \times 1200$ & 112915.37 & +285755.8 & 19.48 & 0.98 & 1.06 & & 5 \\
\hline Pal4-19 (S283) & Feb. 12, 1999 & $1 \times 1080$ & 112915.65 & +285714.7 & 19.53 & 0.95 & 1.08 & & 4 \\
\hline Pal4-21 (S457) & Feb. 11, 1999 & $1 \times 1200$ & 112914.03 & +285845.7 & 19.64 & 0.93 & 1.04 & $\ldots$ & 4 \\
\hline Pal4-23 (S235) & Feb. 12, 1999 & $1 \times 1500$ & 112916.93 & +285806.8 & 19.70 & 0.93 & 1.05 & $\ldots$ & 5 \\
\hline Pal4-24 (S154) & Feb. 11, 1999 & $1 \times 1500$ & 112917.24 & +285746.7 & 19.74 & 0.92 & 1.03 & $\ldots$ & 5 \\
\hline Pal4-25 (S476) & Feb. 12, 1999 & $1 \times 1500$ & 112915.95 & +285847.8 & 19.77 & 0.91 & 1.02 & $\cdots$ & 5 \\
\hline Pal4-26 (S265) & Feb. 12, 1999 & $1 \times 1500$ & 112917.32 & +2858 12.8 & 19.83 & 0.91 & 1.02 & $\ldots$ & 5 \\
\hline Pal4-28 (S426) & Feb. 12, 1999 & $1 \times 1500$ & 112918.50 & +285841.0 & 19.87 & 0.91 & 1.02 & $\ldots$ & 4 \\
\hline Pal4-30 (S276) & Mar. 10, 1999 & $1 \times 1800$ & 112908.80 & +285813.1 & 19.89 & 0.90 & 1.02 & & 5 \\
\hline Pal4-31 (S315) & Feb. 12, 1999 & $1 \times 1500$ & 112916.82 & +285821.5 & 19.89 & 0.93 & 1.03 & $\ldots$ & 5 \\
\hline
\end{tabular}

Notes. ${ }^{(a)}$ IDs preceded by "S" are cross-identifications from Table 7 of Saha et al. (2005). ${ }^{(b)}$ Likely AGB stars.

Table 2. Linelist. "HFS" indicates that hyperfine splitting was taken into account for these transitions.

\begin{tabular}{|c|c|c|c|c|c|c|c|c|c|}
\hline Element & $\begin{array}{c}\lambda \\
{[\AA]}\end{array}$ & $\begin{array}{l}\text { E.P. } \\
{[\mathrm{eV}]}\end{array}$ & $\log g f$ & $\begin{array}{r}E W[\mathrm{~mA}] \\
(\mathrm{RGB})\end{array}$ & Element & $\begin{array}{c}\lambda \\
{[\AA ̊]}\end{array}$ & $\begin{array}{l}\text { E.P. } \\
{[\mathrm{eV}]}\end{array}$ & $\log g f$ & $\begin{array}{r}E W[\mathrm{~mA}] \\
(\mathrm{RGB})\end{array}$ \\
\hline Mg I & 5528.42 & 4.35 & -0.357 & 177 & Cr I & 5300.75 & 0.98 & -2.120 & 113 \\
\hline Mg I & 5711.09 & 4.33 & -1.728 & 108 & $\mathrm{Cr} \mathrm{I}$ & 5329.14 & 2.91 & -0.064 & 79 \\
\hline $\mathrm{Al} \mathrm{I}$ & 6696.03 & 3.14 & -1.347 & 36 & $\mathrm{Cr} \mathrm{I}$ & 5345.81 & 1.00 & -0.980 & 165 \\
\hline Si I & 5684.48 & 4.95 & -1.650 & 33 & Cr I & 5348.33 & 1.00 & -1.290 & 144 \\
\hline $\mathrm{Si}$ I & 5708.41 & 4.95 & -1.470 & 88 & $\mathrm{Cr} \mathrm{I}$ & 6330.09 & 0.94 & -2.914 & 50 \\
\hline $\mathrm{Si}$ I & 5948.55 & 5.08 & -1.230 & 64 & Mn I ${ }^{\mathrm{HFS}}$ & 5394.63 & 0.00 & -3.503 & 166 \\
\hline $\mathrm{Si}$ I & 6142.48 & 5.62 & -0.920 & 22 & Mn I ${ }^{\mathrm{HFS}}$ & 5432.51 & 0.00 & -3.800 & 136 \\
\hline $\mathrm{Si}$ I & 6155.13 & 5.61 & -0.750 & 64 & Mn I $\mathrm{I}^{\mathrm{HFS}}$ & 6013.48 & 3.07 & -0.251 & 102 \\
\hline Ca I & 5261.71 & 2.52 & -0.580 & 107 & $\mathrm{Mn} \mathrm{I}^{\mathrm{HFS}}$ & 6016.62 & 3.08 & -0.216 & 111 \\
\hline $\mathrm{Ca} \mathrm{I}$ & 5590.13 & 2.52 & -0.570 & 114 & $\mathrm{Mn} \mathrm{I}^{\mathrm{HFS}}$ & 6021.75 & 3.08 & 0.034 & 93 \\
\hline $\mathrm{Ca} \mathrm{I}$ & 5601.29 & 2.53 & -0.520 & 116 & Fe I & 4903.32 & 2.88 & -0.926 & 171 \\
\hline $\mathrm{Ca} \mathrm{I}$ & 5857.46 & 2.93 & 0.230 & 157 & $\mathrm{Fe} \mathrm{I}$ & 4938.82 & 2.88 & -1.077 & 121 \\
\hline $\mathrm{Ca} \mathrm{I}$ & 6166.44 & 2.52 & -1.140 & 103 & $\mathrm{Fe} \mathrm{I}$ & 4939.69 & 0.86 & -3.240 & 142 \\
\hline $\mathrm{Ca} \mathrm{I}$ & 6169.04 & 2.52 & -0.800 & 126 & $\mathrm{Fe} \mathrm{I}$ & 5001.87 & 3.88 & 0.050 & 116 \\
\hline $\mathrm{Ca} \mathrm{I}$ & 6169.56 & 2.52 & -0.480 & 143 & $\mathrm{Fe} \mathrm{I}$ & 5006.12 & 2.82 & -0.662 & 173 \\
\hline $\mathrm{Ca} \mathrm{I}$ & 6455.60 & 2.52 & -1.290 & 95 & $\mathrm{Fe} \mathrm{I}$ & 5028.13 & 3.57 & -1.122 & 84 \\
\hline $\mathrm{Ca} \mathrm{I}$ & 6471.67 & 2.52 & -0.875 & 122 & $\mathrm{Fe} \mathrm{I}$ & 5044.21 & 2.85 & -2.059 & 149 \\
\hline $\mathrm{Ca} \mathrm{I}$ & 6499.65 & 2.52 & -0.820 & 115 & Fe I & 5048.44 & 3.94 & -1.029 & 118 \\
\hline Ca I & 6717.69 & 2.71 & -0.610 & 136 & Fe I & 5060.07 & 0.00 & -5.460 & 147 \\
\hline $\mathrm{Sc}$ II & 5031.02 & 1.36 & -0.260 & 95 & $\mathrm{Fe} \mathrm{I}$ & 5068.77 & 2.94 & -1.041 & 159 \\
\hline $\mathrm{Sc}$ II & 5239.81 & 1.46 & -0.770 & 85 & $\mathrm{Fe} \mathrm{I}$ & 5131.48 & 2.22 & -2.515 & 102 \\
\hline $\mathrm{Sc}$ II & 5669.04 & 1.50 & -1.120 & 78 & $\mathrm{Fe} \mathrm{I}$ & 5145.10 & 2.20 & -2.876 & 106 \\
\hline Sc II & 5684.19 & 1.51 & -1.050 & 77 & $\mathrm{Fe} I$ & 5159.05 & 4.28 & -0.820 & 79 \\
\hline Sc II & 6245.62 & 1.51 & -0.980 & 88 & $\mathrm{Fe} I$ & 5162.28 & 4.18 & 0.020 & 157 \\
\hline Sc II & 6604.60 & 1.36 & -1.480 & 36 & $\mathrm{Fe} \mathrm{I}$ & 5166.28 & 0.00 & -4.123 & 170 \\
\hline Ti I & 4997.10 & 0.00 & -1.722 & 132 & $\mathrm{Fe} \mathrm{I}$ & 5192.35 & 3.00 & -0.421 & 163 \\
\hline Ti I & 4999.51 & 0.83 & 0.140 & 180 & $\mathrm{Fe} \mathrm{I}$ & 5195.48 & 4.22 & -0.002 & 109 \\
\hline Ti I & 5001.01 & 2.00 & -0.052 & 78 & $\mathrm{Fe} \mathrm{I}$ & 5196.08 & 4.26 & -0.451 & 57 \\
\hline Ti I & 5009.65 & 0.02 & -1.900 & 105 & $\mathrm{Fe} \mathrm{I}$ & 5215.19 & 3.27 & -0.871 & 133 \\
\hline Ti I & 5039.96 & 0.02 & -1.170 & 134 & $\mathrm{Fe} \mathrm{I}$ & 5216.28 & 1.61 & -2.150 & 175 \\
\hline Ti I & 5064.65 & 0.05 & -0.985 & 152 & $\mathrm{Fe} \mathrm{I}$ & 5217.39 & 3.21 & -1.070 & 130 \\
\hline Ti I & 5147.48 & 0.00 & -1.876 & 149 & $\mathrm{Fe} I$ & 5225.52 & 0.11 & -4.789 & 175 \\
\hline Ti I & 5152.19 & 0.02 & -1.912 & 134 & Fe I & 5242.49 & 3.62 & -0.967 & 110 \\
\hline
\end{tabular}


Table 2. continued.

\begin{tabular}{|c|c|c|c|c|c|c|c|c|c|}
\hline Element & $\begin{array}{c}\lambda \\
{[\AA]}\end{array}$ & $\begin{array}{l}\text { E.P. } \\
{[\mathrm{eV}]}\end{array}$ & $\log g f$ & $\begin{array}{r}E W[\mathrm{~mA}] \\
(\mathrm{RGB})\end{array}$ & Element & $\begin{array}{c}\lambda \\
{[\AA]}\end{array}$ & $\begin{array}{l}\text { E.P. } \\
{[\mathrm{eV}]}\end{array}$ & $\log g f$ & $\begin{array}{r}E W[\mathrm{~m} \AA] \\
(\mathrm{RGB})\end{array}$ \\
\hline Ti I & 5173.75 & 0.00 & -1.120 & 178 & $\mathrm{Fe} \mathrm{I}$ & 5247.05 & 0.09 & -4.946 & 175 \\
\hline Ti I & 5219.70 & 0.02 & -1.980 & 137 & $\mathrm{Fe} \mathrm{I}$ & 5250.22 & 0.12 & -4.938 & 153 \\
\hline Ti I & 5866.46 & 1.07 & -0.840 & 133 & $\mathrm{Fe} \mathrm{I}$ & 5266.56 & 3.00 & -0.490 & 149 \\
\hline Ti I & 5922.12 & 1.05 & -1.470 & 91 & $\mathrm{Fe} \mathrm{I}$ & 5281.80 & 3.04 & -0.833 & 178 \\
\hline Ti I & 5965.83 & 1.88 & -0.410 & 120 & $\mathrm{Fe} \mathrm{I}$ & 5302.31 & 3.28 & -0.720 & 160 \\
\hline Ti I & 6064.63 & 1.05 & -1.970 & 65 & $\mathrm{Fe} \mathrm{I}$ & 5307.37 & 1.61 & -2.987 & 141 \\
\hline Ti I & 6126.22 & 1.07 & -1.420 & 90 & $\mathrm{Fe} \mathrm{I}$ & 5339.94 & 3.27 & -0.720 & 136 \\
\hline Ti I & 6258.10 & 1.44 & -0.355 & 105 & $\mathrm{Fe} \mathrm{I}$ & 5369.97 & 4.37 & 0.536 & 141 \\
\hline Ti I & 6556.08 & 1.46 & -0.943 & 57 & $\mathrm{Fe} \mathrm{I}$ & 5379.57 & 3.68 & -1.514 & 88 \\
\hline Ti I & 6743.13 & 0.90 & -1.630 & 90 & $\mathrm{Fe} \mathrm{I}$ & 5389.49 & 4.42 & -0.410 & 108 \\
\hline Ti II & 5005.16 & 1.57 & -2.550 & 46 & $\mathrm{Fe} \mathrm{I}$ & 5393.18 & 3.24 & -0.715 & 147 \\
\hline Ti II & 5013.68 & 1.58 & -1.935 & 119 & $\mathrm{Fe} \mathrm{I}$ & 5424.08 & 4.32 & 0.520 & 133 \\
\hline Ti II & 5185.91 & 1.89 & -1.350 & 115 & $\mathrm{Fe} \mathrm{I}$ & 5569.63 & 3.42 & -0.500 & 138 \\
\hline Ti II & 5226.55 & 1.57 & -1.300 & 173 & $\mathrm{Fe} \mathrm{I}$ & 5618.64 & 4.21 & -1.275 & 68 \\
\hline Ti II & 5336.78 & 1.58 & -1.700 & 150 & Fe I & 5753.12 & 4.26 & -0.688 & 83 \\
\hline Ti II & 5396.23 & 1.58 & -2.925 & 36 & $\mathrm{Fe} \mathrm{I}$ & 5763.00 & 4.21 & -0.450 & 92 \\
\hline Ti II & 5418.77 & 1.58 & -1.999 & 69 & $\mathrm{Fe} \mathrm{I}$ & 5862.36 & 4.55 & -0.058 & 108 \\
\hline Ti II & 6606.95 & 2.06 & -2.790 & 50 & $\mathrm{Fe} \mathrm{I}$ & 5909.98 & 3.21 & -2.587 & 100 \\
\hline V I & 6039.72 & 1.06 & -0.651 & 78 & $\mathrm{Fe} \mathrm{I}$ & 5916.25 & 2.45 & -2.834 & 124 \\
\hline V I & 6081.44 & 1.05 & -0.578 & 52 & $\mathrm{Fe} \mathrm{I}$ & 5934.65 & 3.93 & -1.170 & 77 \\
\hline V I & 6135.36 & 1.05 & -0.746 & 68 & Fe I & 5956.71 & 0.86 & -4.605 & 146 \\
\hline V I & 6243.10 & 0.30 & -0.978 & 128 & $\mathrm{Fe} \mathrm{I}$ & 5976.78 & 3.94 & -1.310 & 51 \\
\hline V I & 6251.83 & 0.29 & -1.342 & 98 & $\mathrm{Fe} \mathrm{I}$ & 6024.06 & 4.55 & -0.120 & 106 \\
\hline V I & 6274.66 & 0.27 & -1.670 & 66 & Fe I & 6027.06 & 4.08 & -1.089 & 66 \\
\hline Cr I & 5247.57 & 0.96 & -1.640 & 146 & $\mathrm{Fe} \mathrm{I}$ & 6056.01 & 4.73 & -0.460 & 84 \\
\hline Cr I & 5296.70 & 0.98 & -1.400 & 146 & Fe I & 6065.48 & 2.61 & -1.530 & 161 \\
\hline Fe I & 6078.49 & 4.79 & -0.424 & 81 & Fe II & 6432.68 & 2.89 & -3.708 & 23 \\
\hline Fe I & 6137.00 & 2.20 & -2.950 & 146 & Fe II & 6516.08 & 2.89 & -3.380 & 43 \\
\hline Fe I & 6173.34 & 2.22 & -2.880 & 155 & $\mathrm{Co} \mathrm{I}^{\mathrm{HFS}}$ & 5301.01 & 1.71 & -2.000 & 72 \\
\hline Fe I & 6180.21 & 2.73 & -2.586 & 100 & $\mathrm{Co} \mathrm{I}^{\mathrm{HFS}}$ & 5483.31 & 1.71 & -1.488 & 111 \\
\hline Fe I & 6213.44 & 2.22 & -2.481 & 142 & $\mathrm{Co} \mathrm{I}^{\mathrm{HFS}}$ & 6814.89 & 1.96 & -1.900 & 86 \\
\hline Fe I & 6219.29 & 2.20 & -2.448 & 137 & Ni I & 5035.36 & 3.63 & 0.290 & 96 \\
\hline Fe I & 6229.23 & 2.83 & -2.805 & 68 & Ni I & 5080.53 & 3.65 & 0.134 & 75 \\
\hline Fe I & 6232.64 & 3.65 & -1.223 & 115 & $\mathrm{Ni} I$ & 5084.09 & 3.68 & 0.034 & 111 \\
\hline Fe I & 6240.65 & 2.22 & -3.173 & 91 & Ni I & 5146.48 & 3.71 & -0.060 & 70 \\
\hline $\mathrm{Fe} I$ & 6246.32 & 3.60 & -0.733 & 98 & Ni I & 5578.71 & 1.68 & -2.641 & 130 \\
\hline Fe I & 6252.56 & 2.40 & -1.687 & 141 & $\mathrm{Ni} I$ & 5587.85 & 1.94 & -2.142 & 96 \\
\hline Fe I & 6254.25 & 2.28 & -2.443 & 145 & Ni I & 5592.26 & 1.95 & -2.588 & 80 \\
\hline Fe I & 6265.14 & 2.18 & -2.550 & 135 & Ni I & 6128.97 & 1.68 & -3.390 & 73 \\
\hline $\mathrm{Fe} \mathrm{I}$ & 6270.23 & 2.86 & -2.000 & 96 & Ni I & 6176.82 & 4.09 & -0.430 & 52 \\
\hline $\mathrm{Fe} \mathrm{I}$ & 6271.28 & 3.33 & -2.703 & 53 & Ni I & 6177.25 & 1.83 & -3.600 & 48 \\
\hline $\mathrm{Fe} \mathrm{I}$ & 6322.69 & 2.59 & -2.426 & 139 & Ni I & 6327.59 & 1.68 & -3.090 & 97 \\
\hline Fe I & 6335.34 & 2.20 & -2.177 & 141 & Ni I & 6378.26 & 4.15 & -0.820 & 40 \\
\hline Fe I & 6336.83 & 3.69 & -0.856 & 136 & $\mathrm{Ni} I$ & 6482.81 & 1.94 & -2.630 & 84 \\
\hline Fe I & 6344.15 & 2.43 & -2.923 & 119 & Ni I & 6586.32 & 1.95 & -2.812 & 91 \\
\hline Fe I & 6355.03 & 2.84 & -2.350 & 84 & Ni I & 6767.78 & 1.83 & -2.170 & 121 \\
\hline $\mathrm{Fe} \mathrm{I}$ & 6358.69 & 0.86 & -4.468 & 147 & $\mathrm{Cu} \mathrm{I}^{\mathrm{HFS}}$ & 5105.51 & 1.39 & -1.505 & 90 \\
\hline Fe I & 6400.00 & 3.60 & -0.520 & 128 & $\mathrm{Cu} \mathrm{I}^{\mathrm{HFS}}$ & 5782.06 & 1.64 & -1.720 & 79 \\
\hline Fe I & 6400.31 & 0.91 & -3.897 & 168 & Y II & 4883.68 & 1.08 & 0.071 & 110 \\
\hline Fe I & 6475.63 & 2.56 & -2.941 & 103 & Y II & 4900.11 & 1.03 & -0.090 & 115 \\
\hline Fe I & 6481.88 & 2.28 & -2.960 & 130 & Y II & 5087.42 & 1.08 & -0.156 & 84 \\
\hline Fe I & 6498.95 & 0.96 & -4.687 & 144 & Y II & 5200.41 & 0.99 & -0.570 & 98 \\
\hline $\mathrm{Fe} \mathrm{I}$ & 6518.37 & 2.83 & -2.450 & 105 & Y II & 5509.90 & 0.99 & -1.015 & 93 \\
\hline $\mathrm{Fe} \mathrm{I}$ & 6574.22 & 0.99 & -5.004 & 115 & Zr II & 5112.28 & 1.66 & -0.590 & 42 \\
\hline $\mathrm{Fe} \mathrm{I}$ & 6581.21 & 1.48 & -4.680 & 57 & Ba II & 4554.03 & 0.00 & 0.170 & 274 \\
\hline $\mathrm{Fe} \mathrm{I}$ & 6609.12 & 2.56 & -2.692 & 128 & Ba II & 5853.00 & 0.60 & -1.010 & 130 \\
\hline Fe I & 6739.52 & 1.56 & -4.794 & 59 & Ba II & 6141.73 & 0.70 & -0.077 & 212 \\
\hline $\mathrm{Fe} \mathrm{I}$ & 6750.15 & 2.42 & -2.608 & 140 & Ba II & 6496.91 & 0.60 & -0.380 & 201 \\
\hline $\mathrm{Fe}$ II & 4923.93 & 2.89 & -1.307 & 166 & La II & 5114.56 & 0.23 & -1.060 & 55 \\
\hline $\mathrm{Fe}$ II & 4993.35 & 2.81 & -3.485 & 37 & La II & 6390.46 & 0.32 & -1.400 & 45 \\
\hline $\mathrm{Fe}$ II & 5197.58 & 3.23 & -2.233 & 88 & Ce II & 5274.23 & 1.04 & 0.150 & 33 \\
\hline $\mathrm{Fe}$ II & 5234.63 & 3.22 & -2.220 & 94 & Nd II & 5249.59 & 0.98 & 0.217 & 54 \\
\hline $\mathrm{Fe}$ II & 5425.26 & 3.20 & -3.372 & 22 & Dy II & 5169.69 & 0.10 & -1.660 & 12 \\
\hline $\mathrm{Fe}$ II & 6247.56 & 3.89 & -2.329 & 24 & & & & & \\
\hline
\end{tabular}

